\title{
THE $G$ AND $H$ FUNCTIONS AS SYMMETRICAL FOURIER KERNELS
}

\author{
BY \\ CHARLES FOX
}

1. Introduction. The functions $k(x)$ and $h(x)$ are said to form a pair of Fourier kernels if the following pair of reciprocal equations are simultaneously valid:

$$
\begin{aligned}
& g(x)=\int_{0}^{\infty} k(x y) f(y) d y, \\
& f(x)=\int_{0}^{\infty} h(x y) g(y) d y .
\end{aligned}
$$

The kernels are said to be symmetrical if $k(x)=h(x)$ and unsymmetrical if $k(x) \neq h(x)$. The symmetrical case only will concern us here.

Various sets of conditions have been discovered which ensure the validity of (1), (2), the set we use here consists of convergence conditions on $f(x)$, $k(x)$ and $h(x)$ together with a functional equation satisfied by the Mellin transforms of $k(x)$ and $h(x) . K(s)$ is said to be the Mellin transform of $k(x)$ if

$$
K(s)=\int_{0}^{\infty} k(x) x^{s-1} d x .
$$

If $H(s)$ is the Mellin transform of $h(x)$ then the functional equation in question is

$$
K(s) H(1-s)=1,
$$

[6, p. $214(8.3 .5)]$.

Our first aim is to find the most general $G$-function which is also a symmetrical Fourier kernel. The $G$-function is a sum of hypergeometric functions each of which is usually an entire function. It is easily defined by means of its Mellin transform so that the satisfaction of the functional equation (4) is quickly determined. The $G$-function has been much studied by many authors but these studies have been mainly confined to establishing the differential equations they satisfy, the linear relationships which exist between them and the various asymptotic forms they assume for large values of the variable. In particular Barnes [1] and Meijer [4] have written lengthy memoirs on the asymptotic problem. Here I shall concentrate entirely on the $G$-function as a symmetrical Fourier kernel.

Presented to the Society, April 16, 1960; received by the editors January 11, 1960. 
Following Meijer's definition [4, p. 229], but with $s$ replaced by $-s$, the $G$-function is written as on the left of (5) below and defined by the integral on the right:

$$
G_{p, q}^{m, n}\left(x \mid \begin{array}{l}
a_{1}, \cdots, a_{p} \\
b_{1}, \cdots, b_{q}
\end{array}\right)=\frac{1}{2 \pi i} \int_{T} \frac{\prod_{i=1}^{m} \Gamma\left(b_{i}+s\right) \prod_{i=1}^{n} \Gamma\left(1-a_{i}-s\right)}{\prod_{i=m+1}^{q} \Gamma\left(1-b_{i}-s\right) \prod_{i=n+1}^{p} \Gamma\left(a_{i}+s\right)} x^{-s} d s .
$$

The poles of the integrand must be simple and those of $\Gamma\left(b_{i}+s\right), i=1, \cdots, m$, must lie on one side of the contour $T$ and those of $\Gamma\left(1-a_{i}-s\right), i=1, \cdots, n$, must lie on the other side.

If $M(s)$ denotes the coefficient of $x^{-s}$ in the integrand of (5) then evidently $M(s)$ is the Mellin transform of the $G$-function on the left of (5). It is therefore easy to decide whether the functional equation

$$
M(s) M(1-s)=1
$$

is or is not satisfied. The satisfaction of (6) alone, however, is insufficient to ensure the validity of the reciprocity (1), (2). More is required, but if (6) is established then much progress has been made towards the setting up of (1), (2).

I shall prove here that the following $G$-function, denoted by $G(x)$ for brevity,

$$
G(x)=\frac{1}{c} G_{2 p, 2 q}^{q, p}\left(\begin{array}{l|l}
x^{1 / c} & \begin{array}{l}
a_{1}, \cdots, a_{p}, 1-c-a_{1}, \cdots, 1-c-a_{p} \\
b_{1}, \cdots, b_{q}, 1-c-b_{1}, \cdots, 1-c-b_{q}
\end{array}
\end{array}\right),
$$

is a symmetrical Fourier kernel. The reciprocity (1), (2) will be established in two cases; in the first case we shall use convergence in mean square and in the second we shall use the methods of ordinary convergence. As is usual in Fourier transform theory the second case is much more difficult.

If (7) is a symmetrical Fourier kernel then it is easy to deduce that [6, p. 220]

$$
\frac{k^{1 / 2}}{c} G_{2 p, 2 q}^{q, p}\left((k x)^{1 / c} \mid \begin{array}{l}
a_{1}, \cdots a_{p}, 1-c-a_{1}, \cdots, 1-c-a_{p} \\
b_{1}, \cdots b_{q}, 1-c-b_{1}, \cdots, 1-c-b_{q}
\end{array}\right)
$$

is also a symmetrical Fourier kernel. The function (8) seems to contain most of the well-known Fourier kernels when $c=1 / 2$. When $c=k=1 / 2, q=1$ and $p=0$ the function (8) becomes a Bessel function. When $c=k=1 / 2, q=2$ and $p=0$ then (8) gives us Fourier kernels which are frequently products of Bessel functions.

In a previous paper [3] I have given some general theorems for the kernels of (1), (2). In Theorem 2 of that paper [3, p. 407] it is possible to choose the first kernel so as to be equal to 


$$
2^{1 / 2} G_{2 p, 2 p+2}^{p+1, p}\left(\frac{x^{2}}{4}\left(\begin{array}{l}
a_{1}, \cdots, a_{p}, \frac{1}{2}-a_{1}, \cdots, \frac{1}{2}-a_{p} \\
b_{1}, \cdots, b_{p+1}, \frac{1}{2}-b_{1}, \cdots, \frac{1}{2}-b_{p+1}
\end{array}\right) .\right.
$$

With this choice of the first kernel the second kernel of the theorem also becomes equal to (9) and the theorem then specializes to the case of the symmetrical kernel. Evidently (9) is a special case of (8) with $c=k=1 / 2$ and $q=p+1$. As a final example, again for the case $c=1 / 2$, Roop Narain [5] proves that the Mellin transform of (8) satisfies (6) when $c=1 / 2$. He does not, however, proceed to establish the reciprocities (1), (2).

The rest of the contents of this paper are briefly as follows. I shall first establish the reciprocities (1), (2) with $G(x)$ of (7) as the symmetrical Fourier kernel. I confine myself to (7) because the Mellin transforms of $G$-functions which are not included in the type (7) do not satisfy (6) and therefore cannot be Fourier kernels. The formulae (1), (2) will be set up in two cases, using convergence in mean square methods in the first case and ordinary convergence methods in the second. As frequently happens in Fourier transform theory the second case is much more difficult. After setting up (1), (2) I shall state and prove a general formula for the self reciprocal functions associated with $G(x)$.

In the second part of this paper I shall define a function $H(x)$ which is much more general than $G(x)$ of (7). I shall then establish (1), (2) with $H(x)$ as the kernel and also give a formula for the self reciprocal functions associciated with $H(x)$. Finally I shall obtain the asymptotic expansion of $H(x)$ when $x$ is real and positive, using a method which I originated in my previous paper on Fourier kernels [3].

2. Some properties of $G(x)$. Using the definition of $G(x)$ given by the Bateman project $[2$, p. 207] the $G(x)$ of $(7)$ is defined by

$$
G(x)=\frac{1}{2 \pi i c} \int_{T_{1}} \frac{\prod_{m=1}^{q} \Gamma\left(b_{m}+s\right) \prod_{n=1}^{p} \Gamma\left(1-a_{n}-s\right)}{\prod_{m=1}^{q} \Gamma\left(c+b_{m}-s\right) \prod_{n=1}^{p} \Gamma\left(1-c-a_{n}+s\right)} x^{-s / c} d s
$$

All poles of the integrand in (10) must be simple and the contour $T_{1}$ must separate the poles so that those of $\Gamma\left(b_{m}+s\right), m=1, \cdots, q$, lie on one side of $T_{1}$ and those of $\Gamma\left(1-a_{n}-s\right), n=1, \cdots, p$, lie on the other side of $T_{1}$. For reasons which appear later we shall assume that $q \geqq p+1$ so that $q$ can never take the value 0 . We do, however, allow the value $p=0$ if, when this is the case, the two products in (10) from $n=1$ to $n=p$ are each replaced by unity. Finally we assume $x$ to be real and positive.

On replacing $s$ by $c s$ in (10) we obtain 
(11)

$$
G(x)=\frac{1}{2 \pi i} \int_{T} \frac{\prod_{m=1}^{q} \Gamma\left(b_{m}+c s\right) \prod_{n=1}^{p} \Gamma\left(1-a_{n}-c s\right)}{\prod_{m=1}^{q} \Gamma\left(c+b_{m}-c s\right) \prod_{n=1}^{p} \Gamma\left(1-c-a_{n}+c s\right)} x^{-s} d s
$$

In (11), if $s=\sigma+i t$, we take the contour $T$ to be the line from $\sigma-i \infty$ to $\sigma+i \infty$. In (11) we assume that $c>0$, that all the poles of the integrand are simple and that those of $\Gamma\left(b_{m}+c s\right), m=1, \cdots, q$, lie on the left of $T$ and that those of $\Gamma\left(1-a_{n}-c s\right), n=1, \cdots, p$, lie on the right of $T$.

Let

$$
M(s)=\frac{\prod_{m=1}^{q} \Gamma\left(b_{m}+c s\right) \prod_{n=1}^{p} \Gamma\left(1-a_{n}-c s\right)}{\prod_{m=1}^{q} \Gamma\left(c+b_{m}-c s\right) \prod_{m=1}^{p} \Gamma\left(1-c-a_{n}-c s\right)} .
$$

Evidently $M(s)$ is the Mellin transform of $G(x)$, in the sense inverse to that of (3), and

$$
M(s) M(1-s)=1 .
$$

For large $s$ the asymptotic expansion of the Gamma function is [7, p. 272]

$$
\log \Gamma(s+a)=\left(s+a-\frac{1}{2}\right) \log s-s+\frac{1}{2} \log (2 \pi)+A s^{-1}+O\left(s^{-2}\right),
$$

where $|\arg s| \leqq \pi-\delta, \delta>0, A$ is a constant independent of $s$ and $O$ is the Landau order symbol. Along the line $T$ we write $s=\sigma+i t$ ( $\sigma$ and $t$ real) and in $M(s)$ we replace Gamma functions involving - cs by Gamma functions containing $+c s$ with the help of the formula $\Gamma(z) \Gamma(1-z)=\pi / \sin (\pi z)$, [7, p. 233]. For brevity we write $h=2 c(q-p)$ (for reasons which appear shortly $h$ will be positive) and then from (14) along the line $T$ for large positive or negative $t$ we have

$$
M(s) x^{-s}=|t|^{h(\sigma-1 / 2)} \exp \{i t(h \log t-\log x+B)\}\left\{D+O\left(|t|^{-1}\right)\right\}
$$

where $B$ is a constant and $D$ is also a constant but $D$ may have one value for large positive $t$ and another value for large negative $t$.

On a large circle whose points have the polar co-ordinates $(R, \theta)$ it follows from (14) that $M(s)$ is dominated by the factor $\exp \{(h R \log R) \cos \theta\}$. Hence if $h$ is positive the contour $T$ can be closed by a large semicircle on the left while if $h$ is negative it must be closed by a large semicircle on the right. Consequently if $h>0$ the residues of the integrand of (11) at the poles of 
$\Gamma\left(b_{m}+c s\right), m=1, \cdots, q$, can be used to express $G(x)$ in terms of hypergeometric functions. These functions are series of positive powers of $x$ and entire functions. But when $h<0$ we must use the poles of $\Gamma\left(1-a_{n}-c s\right)$, $n=1, \cdots, p$, and these lead to an expression for $G(x)$ in negative powers of $x$. Since such series present special difficulties which I do not wish to discuss here I shall assume for the rest of this paper that $h>0$. Now $c>0$ and $q$ and $p$ are positive integers or zero, hence $h=2 c(q-p)>0$ implies that $q \geqq p+1$ and this is the way in which the assumption $h>0$ will be written.

From (15) the integral of (11) is uniformly convergent with respect to $x$ when $\sigma<1 / 2$. On integrating through the integral sign we then obtain from (11)

$$
G_{1}(x)=\int_{0}^{x} G(x) d x
$$

and

$$
G_{1}(x)=\frac{1}{2 \pi i} \int_{T} \frac{M(s)}{1-s} x^{1-8} d s
$$

We have proved (16) and (17) for the case $\sigma<1 / 2$ but the case of interest to us is when $\sigma=1 / 2$ because the line $\sigma=1 / 2$ is the contour of Theorem 1 in the next section. We can also prove that (16) and (17) are true when $\sigma=1 / 2$ by the following methods. On multiplying (15) by $1 / t$ we see that the integral of (17) converges when $\sigma=1 / 2$ and, since $h>0$, that we may close the contour by a large semicircle on the left. If, as is the case in Theorem 1, all the poles of $\Gamma\left(b_{m}+c s\right), m=1, \cdots, q$, lie on the left of $\sigma=1 / 2$ and all other poles lie on the right then the computation of the integral in (17) by residues gives us quite easily the individual powers of $G(x)$ each integrated from 0 to $x$. Since $G(x)$ is an entire function such term by term integration is justified and the final result is $G_{1}(x)$ as defined by (16). Hence, with the conditions of Theorem $1,(16)$ and (17) are true when $\sigma=1 / 2$.

Again with $s=\sigma+i$ as before we see from (15) that on the line $\sigma=1 / 2$ the function $M(s) x^{-s}$, as a function of $s$, is bounded for all values of $t$. Hence on $\sigma=1 / 2, M(s) x^{-s} /(1-s) \in L_{2}(1 / 2-i \infty, 1 / 2+i \infty)$ and so the integral of (17) converges in mean square. But, with the conditions of Theorem 1 as proved in the next section, $\S 3$, the integral in (17) also converges in the ordinary sense to $G_{1}(x)$ of $(16)$. Hence if this integral is evaluated by mean square methods its value will also be $G_{1}(x)$ of (17) except, perhaps, in a set of measure zero.

This property of (17) enables us to prove Theorem 1.

3. Convergence in mean square.

THEOREM 1. $\operatorname{Re}(z)$ denotes the real part of $z$. If (i) in $M(s)$ of (12) we have 
$c>0, q \geqq p+1, \operatorname{Re}\left(b_{m}\right)>-c / 2, m=1, \cdots, q$, and $\operatorname{Re}\left(a_{n}\right)<(2-c) / 2$, (ii) $f(x) \in L_{2}(0, \infty)$ then

$$
g(x)=\frac{d}{d x} \int_{0}^{\infty} G_{1}(x u) f(u) \frac{d u}{u}
$$

defines, almost everywhere, a function $g(x) \in L_{2}(0, \infty)$,

$$
f(x)=\frac{d}{d x} \int_{0}^{\infty} G_{1}(x u) g(u) \frac{d u}{u}
$$

holds almost everywhere and

$$
\int_{0}^{\infty}\{f(x)\}^{2} d x=\int_{0}^{\infty}\{g(x)\}^{2} d x .
$$

The proof of this theorem is based upon Theorem 129, Titchmarsh [6, p. 222].

In (17), which defines the relation between $G_{1}(x)$ and $M(s)$ (the Mellin transform of $G(x)$ ), we take $\sigma=1 / 2,(s=\sigma+i t)$ so that the contour $T$ is the line $1 / 2-i \infty$ to $1 / 2+i \infty$. The inequalities (i) then ensure that all the poles of $\Gamma\left(b_{m}+c s\right), m=1, \cdots, q$, lie on the left and those of $\Gamma\left(1-a_{n}-c s\right)$ lie on the right of $T$, as required for the definition of $G(x)$.

We now proceed to establish the truth of the three requirements of Theorem 129 cited above.

The first requirement is that $M(1 / 2+i t) M(1 / 2-i t)=1$, and this is true because of (13). The second requirement is that $G_{1}(x)$ and $M(s)$ should be related as in (17) and the third that $f(x) \in L_{2}(0, \infty)$, which is covered by (ii).

Since all the conditions of Theorem 129 [6, p. 221] are satisfied the conclusions of that theorem follow and for our case these conclusions are (a), (b) and (c) above.

This completes the proof of Theorem 1.

4. The case of ordinary convergence. We now proceed to set up the reciprocities (1), (2) for the much more difficult case of ordinary convergence. The proof is based upon Titchmarsh, Theorem 134 [6, p. 232].

TheOREM 2. Let $h=2 c(q-p)$ and let $\operatorname{Re}(z)$ denote the real part of $z$. If

(i) $c>0, q \geqq p+1$, (so that $h>0$ ),

(ii) $\operatorname{Re}\left(b_{m}\right) \geqq c(1-h) / 2 h, m=1, \cdots, q$, $\operatorname{Re}\left(a_{n}\right)<(2 h-c h-c) / 2 h, n=1, \cdots, p$,

(iii) $f(y) y^{(1-h) / 2 h} \in L(0, \infty)$ and $f(y)$ is of bounded variation near $y=x$, $(x>0)$, then

$$
\int_{0}^{\infty} G(x u)\left\{\int_{0}^{\infty} G(u y) f(y) d y\right\} d u=\frac{1}{2}\{f(x+0)+f(x-0)\} .
$$


We commence the proof by making the following transformations,

$$
x=X^{h}, \quad y=Y^{h} \text { and } u=(U c / h)^{h},
$$

in (18). Since $y$ and $u$ are variables of integration we have, after some obvious adjustments for $Y$ and the parameter $X$

$$
\begin{aligned}
& \int_{0}^{\infty} h\left(\frac{c}{h}\right)^{h / 2} G\left(\left\{\frac{X U c}{h}\right\}^{h}\right)(X U)^{(h-1) / 2} \\
& \cdot\left[\int_{0}^{\infty} h\left(\frac{c}{h}\right)^{h / 2} G\left(\left\{\frac{U Y c}{h}\right\}^{h}\right)(U Y)^{(h-1) / 2} f\left(Y^{h}\right)\left(\frac{Y}{X}\right)^{(h-1) / 2} d Y\right] d U \\
& =\frac{1}{2}\left\{f\left(X^{h}+0\right)+f\left(X^{h}-0\right)\right\} .
\end{aligned}
$$

Evidently the kernel $G(x)$ has been replaced by the kernel

$$
h\left(\frac{c}{h}\right)^{h / 2} G\left(\left\{\frac{X c}{h}\right\}^{h}\right) X^{(h-1) / 2} .
$$

From (11) we have

$$
\begin{array}{r}
h\left(\frac{c}{h}\right)^{h / 2} G\left(\left\{\frac{X c}{h}\right\}^{h}\right) X^{(h-1) / 2} \\
=\frac{1}{2 \pi i} \int \frac{\prod_{m=1}^{q} \Gamma\left(b_{m}+c s\right) \prod_{n=1}^{p} \Gamma\left(1-a_{n}-c s\right)}{\prod_{m=1}^{q} \Gamma\left(c+b_{m}-c s\right) \prod_{n=1}^{p} \Gamma\left(1-a_{n}-c-c s\right)} \\
\cdot\left(\frac{X c}{h}\right)^{(-2 s h+h-1) / 2}(c h)^{1 / 2} d s,
\end{array}
$$

where the contour of integration is a straight line parallel to the imaginary axis in the $s$ plane. The contour must lie in a strip of width $1 / 2 h$ with its right hand boundary to the right of the imaginary $s$ axis and distance $1 / 2$ from it.

We now make one more transformation

$$
2 s h-h+1=2 S .
$$

Equation (21) then becomes

$$
h\left(\frac{c}{h}\right)^{h / 2} G\left(\left\{\frac{X c}{h}\right\}^{h}\right) X^{(h-1) / 2}=\frac{1}{2 \pi i} \int K(S) X^{-S} d S,
$$

where 


$$
\begin{aligned}
K(S)=\frac{\prod_{m=1}^{q} \Gamma\left(b_{m}+\frac{c}{2}-\frac{c}{2 h}+\frac{c S}{h}\right) \prod_{n=1}^{p} \Gamma\left(1-a_{n}-\frac{c}{2}+\frac{c}{2 h}-\frac{c S}{h}\right)}{\prod_{m=1}^{q} \Gamma\left(b_{m}+\frac{c}{2}+\frac{c}{2 h}-\frac{c S}{h}\right) \prod_{n=1}^{p} \Gamma\left(1-a_{n}-\frac{c}{2}-\frac{c}{2 h}+\frac{c S}{h}\right)} \\
\cdot\left(\frac{c}{h}\right)^{1 / 2-S} \cdot
\end{aligned}
$$

If $S=\sigma+i t$, then the contour for (23) is any line from $\sigma_{0}-i \infty$ to $\sigma_{0}+i \infty$ where $0<\sigma_{0}<1 / 2$.

As a consequence of these transformations we can now proceed to establish (20) from (24) by using Theorem 134 [6, p. 232]. This theorem is based upon six conditions, four of which apply to $K(S)$ and two to $f\left(Y^{h}\right)$, and we now prove that all these conditions are fulfilled.

Let $S=\sigma+i t$; then the first condition is that we can find $\sigma_{0}<0$ and $\sigma_{1}>1$ such that $K(S)$ is regular in the strip $\sigma_{0}<\sigma<\sigma_{1}$, except possibly for a finite number of simple poles on the imaginary axis, $\sigma=0$. To prove that this is true let $\Sigma$ denote the strip $S=\sigma+i t, 0 \leqq \sigma \leqq 1$ and $-\infty<t<\infty$. From (24) the poles of $K(S)$ are evidently at the points

$$
-\frac{r h}{c}-\frac{h}{c}\left(b_{m}-\frac{c(1-h)}{2 h}\right), \quad \frac{r h}{c}+\frac{h}{c}\left(1-a_{n}+\frac{c(1-h)}{2 h}\right)
$$

where $r$ is a positive integer or zero. If in condition (ii) above only the inequalities hold then it is evident that all the $a_{n}$ poles lie to the right of $\Sigma$ and all of the $b_{m}$ poles lie to the left of $\Sigma$, none being on the boundaries of $\Sigma$. If, however, some of the equalities for $b_{m}$ hold in (ii) then there are some simple poles of $K(S)$ on the imaginary axis and there can be at most $q$ of these.

Finally since the only singularities of $K(S)$ are isolated simple poles it follows that $\sigma_{0}<0$ and $\sigma_{1}>1$ can be found so that $K(S)$ is regular in the strip $\sigma_{0}<\sigma<\sigma_{1}$ except for a finite number of simple poles on the imaginary axis.

The second condition, which is the most difficult of the six, requires an investigation into the asymptotic expansion of $K(S)$ for $S=\sigma+i$ and large positive or negative values of $t$. The formula $\Gamma(z) \Gamma(1-z)=\pi / \sin (\pi z),[7$, p. 233] is first used in order to convert those Gamma functions containing $-c S / h$ into Gamma functions containing $+c S / h$. The asymptotic expansion for the Gamma function given by (14) is now applied to

$$
\frac{\Gamma\left(b_{m}+\frac{c}{2}-\frac{c}{2 h}+\frac{c S}{h}\right)}{\Gamma\left(b_{m}+\frac{c}{2}+\frac{c}{2 h}-\frac{c S}{h}\right)} .
$$

It is then found that for large $S,|\arg S|\langle\pi-\delta, \delta>0$, the asymptotic ex- 
pansion of (25) is the product of the following two factors (here $O$ denotes the Landau order symbol):

$$
\left(\frac{c}{h}\right)^{c(2 S-1) / h} \exp \left\{\frac{2 c}{h}\left[\left(S-\frac{1}{2}\right) \log S-S\right]\right\}
$$

and

$$
\left(B_{m}+\frac{D_{m}}{S}+O\left(\frac{1}{\left|S^{2}\right|}\right)\right) \sin \pi\left(b_{m}+\frac{c}{2}+\frac{c}{2 h}-\frac{c S}{h}\right) .
$$

These factors will occur in the numerator of the asymptotic expansion for $K(S)$ and, since $m=1, \cdots, q$, there will be $q$ such factors in the numerator.

On applying the same analysis to

$$
\frac{\Gamma\left(1-a_{n}-\frac{c}{2}+\frac{c}{2 h}-\frac{c S}{h}\right)}{\Gamma\left(1-a_{n}-\frac{c}{2}-\frac{c}{2 h}+\frac{c S}{h}\right)}
$$

we see that owing to the change in sign of $S$ the factors will now be in the denominator of the asymptotic expansion of $K(S)$. Since $n=1, \cdots, p$ there will then be $p$ factors in the denominator of $K(S)$ identical with (27) and another $p$ factors similar to (28), the main change in (28) being that $b_{m}+c / 2$ must be replaced by $1-a_{n}-c / 2$ and the constants $B_{m}$ and $D_{m}$ must be replaced by other constants.

Dealing first with the factors (27) the total effect is that in the asymptotic expansion of $K(S)$ the terms of (27) will appear raised to the power $(q-p)$. Noting that $h=2 c(q-p)$ the result of raising $(27)$ to the power $(q-p)$ is

$$
\begin{aligned}
\left(\frac{c}{h}\right)^{(S-1 / 2)} \exp & \left\{\left(S-\frac{1}{2}\right) \log S-S\right\} \\
= & \left(\frac{c}{h}\right)^{(S-1 / 2)}\left\{A+\frac{B}{S}+O\left(\frac{1}{S^{2}}\right)\right\} \Gamma(S)
\end{aligned}
$$

on using the asymptotic expansion for $\Gamma(S)$, where $A$ and $B$ are constants.

We now consider the contribution to the asymptotic expansion of $K(S)$ of terms such as given by (28). On putting $S=\sigma+i t$ in (31) below and allowing $t$ to become large and either positive or negative it is evident that

$$
\frac{\sin \pi\left(b_{m}+\frac{c}{2}+\frac{c}{2 h}-\frac{c S}{h}\right)}{\left(\cos \frac{1}{2} S \pi\right)^{2 c / h}}=C+O\left(e^{-|\ell|}\right)
$$


where $C 2^{-(2 c-h) / h}$ has the value $-i \exp \left\{i\left(b_{m}+c(h+1)\right) / 2 h\right\}$ for large positive values of $t$ and $+i \exp \left\{-i\left(b_{m}+c(h+1)\right) / 2 h\right\}$ for large negative values of $t$. The form of (31) still holds if $-c S / h$ is replaced by $+c S / h$ which occurs when the $a_{n}$ factors are considered.

We can now assess the total contribution of factors such as (28) to the asymptotic expansion of $K(S)$ when $S=\sigma+i t$ and $t$ is large. Since there are $q$ such factors in the numerator and $p$ in the denominator and also $h=2 c(q-p)$ their total contribution will be

$$
\left\{D+\frac{E}{S}+O\left(\frac{1}{\left|S^{2}\right|}\right)\right\} \cos \left(\frac{1}{2} S \pi\right),
$$

where the constants $D$ and $E$ each have one value when $t$ is large and positive and, perhaps, another value when $t$ is large and negative. From (24) the required asymptotic expansion is then the product of (30), (32) and the factor $(c / h)^{1 / 2-S}$. The final result we have established is therefore as follows: for $S=\sigma+i t$ and sufficiently large values of $t$ the asymptotic expansion of $K(S)$ is given by

$$
K(S)=\Gamma(S) \cos \left(\frac{1}{2} S \pi\right)\left\{\alpha+\frac{\beta}{S}+O\left(\frac{1}{\left|S^{2}\right|}\right)\right\},
$$

where the constants $\alpha$ and $\beta$ each have one value when $t$ is large and positive and, perhaps, another value when $t$ is large and negative.

Equation (33) shows that the second condition of Theorem 134 [6, p. 232] is fulfilled.

The remaining four conditions of this theorem are comparatively easy to discuss. The third condition is that

$$
K(S) K(1-S)=1 \text {, }
$$

and this is easily established from (24). The fourth condition is that

$$
h\left(\frac{c}{h}\right)^{h / 2} G\left(\left\{\frac{X c}{h}\right\}^{h}\right) X^{(h-1) / 2}
$$

must be the Mellin transform of $K(S)$. This, of course, is evident from (23).

The fifth and sixth conditions are that

$$
\int_{0}^{\infty}\left|f\left(Y^{h}\right) Y^{(h-1) / 2}\right| d Y
$$

must exist and that the integrand of (34) must be of bounded variation near $Y=X,(X>0)$. On writing $Y^{h}=y$ it follows from (iii), in the enunciation of Theorem 2, that these two conditions are also fulfilled.

Since we have proved that all the conditions of Theorem 134 [6, p. 232] are satisfied it follows that the conclusions of this theorem are true and, con- 
sequently, that (20) above is true. On using the transformations (19) in reverse the truth of (18) follows and the proof of Theorem 2 is then completed.

5. Some properties of $G(x)$. In the previous section we proved that $G(x)$ is a symmetrical Fourier kernel by first transforming it and then proving that the transformed function is a symmetrical Fourier kernel. The necessity for such a transformation arises for the following reasons.

The classical kernels such as $\sin x, \cos x, x^{1 / 2} J_{m}(x)$ all behave in a very similar manner as $|x| \rightarrow \infty$. When $x$ is complex they all tend to infinity with exponential rapidity but when $x$ is real they all oscillate finitely as $x \rightarrow \infty$, just like $\sin x$. Now it is the behaviour of a function on the real axis which decides whether it is a Fourier kernel or not and so the familiar finite oscillation on the real axis has formed the basis for much classical theory of Fourier kernels, e.g. Theorem 134 [6, p. 232] which has been used in the previous section. The function $G(x)$ of $(7)$ does not, however, necessarily behave in this manner when $x \rightarrow \infty$ through positive values.

The asymptotic expansion for $G(x)$ as defined by (7) is given by Meijer [4, Theorem 18, assertion 4, p. 1065 (p. 1065 , line 4 , for $m+1=p / 2+q / 2$ read $m+n=p / 2+q / 2$ ) and Theorem 9, p. 772]. For large real positive $x$ we have

$$
\begin{aligned}
G(x)= & x^{(1-h) / 2 h}\left[\cos \left\{2(q-p) x^{1 / h}+\alpha\right\}\left\{A+O\left(x^{-2 / h}\right)\right\}\right. \\
& \left.+\sin \left\{2(q-p) x^{1 / h}+\alpha\right\} O\left(x^{-1 / h}\right)\right] \\
& +\sum_{n=1}^{p} x^{-\left(1-a_{n}\right) / c}\left\{P_{n}+O\left(x^{-1 / c}\right)\right\},
\end{aligned}
$$

where, as before, $h=2 c(q-p), O$ is an order symbol for large $x$ and $\alpha, A, P_{n}$ are constants. On writing $x=X^{h}$ and using (ii) Theorem 2

$$
\left(\operatorname{Re}\left(a_{n}\right)<(2 h-c h-c) / 2 h\right)
$$

(35) becomes

(36) $G\left(X^{h}\right) X^{(h-1) / 2}=\cos \{2(q-p) X+\alpha\}\left\{A+O\left(X^{-1}\right)\right\}+O\left(X^{-(1+2 \beta) / 2}\right)$,

where $\beta>1 / 2 h$. All the algebraical terms of (35) in the summation from $n=1$ to $n=p$ have been merged into the term $O\left(X^{-(1+2 \beta) / 2}\right)$. From (36) the kernel defined by (21) oscillates in the familiar manner like $\cos X$ as $X \rightarrow \infty$ through positive values. Consequently we can prove (20) by a direct appeal to Theorem $134[6$, p. 232] whereas (18) cannot be proved in such a manner.

It is now evident that in order to obtain the finite oscillation along the real axis of the kernels in (20) the first $G$-function must be multiplied by $(X U)^{(h-1) / 2}$ and the second by $(U Y)^{(h-1) / 2}$. The transformation (19), $u=(U c / h)^{h}, d u=h U^{(h-1)}(c / h)^{h} d U$, gives us the power of $U$ we require but $y=Y^{h}, d y=h Y^{(h-1)} d Y$ gives us a power $Y^{(h-1) / 2}$ in excess of our requirements. 
This slight difficulty is easily overcome by attaching the excess power to $f\left(Y^{h}\right)$ as is done in (20).

These are the main reasons for the method of proof used in the previous section.

All Fourier kernels are associated with certain discontinuous integrals. The ones associated with $G(x)$ are given by the easily proved theorem below.

THEOREM 3. Let $h$ be defined as in Theorem 2 and $G_{1}(x)$ as in (16). If conditions (i) and (ii) of Theorem 2 hold then

$$
\begin{aligned}
0, & x>y>0, \\
\int_{0}^{\infty} G(x u) \frac{G_{1}(u y)}{u} d u=\frac{1}{2}, & y=x>0, \\
1, & y>x>0 .
\end{aligned}
$$

To prove this we choose $f(y)$ in (18) Theorem 2 so that $f(y)=1$ when $y<Y$ and $f(y)=0$ when $y>Y$. Hence $f(x)=1$ when $x<Y, f(x)=0$ when $x>Y, f(x-0)=1$ when $x=Y$ and $f(x+0)=0$ when $x=Y$. The integral with respect to $y$ in (18) then has 0 and $Y$ for its limits of integration and integrates to $G_{1}(u Y) / u$. The final result is (37) above except that $y$ has been replaced by $Y$.

6. Self reciprocal functions associated with the $G$-function. If

$$
f(x)=\int_{0}^{\infty} f(y) k(x y) d y
$$

then $f(x)$ is said to be a self reciprocal function for the kernel $k(x)$. All symmetrical Fourier kernels can be associated with self reciprocal functions and conversely. Let $K(s)$ be the Mellin transform of $k(x)$ and $F(s)$ that of $f(x)$ as defined by (3). Now multiply (38) by $x^{8-1}$ and integrate with respect to $x$. If the change of order of integration on the right hand side can be justified we easily obtain

$$
F(s)=F(1-s) K(s)
$$

From (39) follows

$$
K(s) K(1-s)=1
$$

which is the functional equation which all symmetrical Fourier kernels must satisfy.

Conversely if $k(x)$ is a symmetrical Fourier kernel its Mellin transform $K(s)$ must satisfy $(40)$ and we may therefore expect $K(s)$ to be expressible in the form

$$
K(s)=F(s) / F(1-s)
$$


If this is the case then by working backwards from (39) to (38) we see that $f(x)$ will be a self reciprocal function for $k(x)$.

The self reciprocal functions for $G(x)$ are given by Theorem 4 below. The following formulae are useful in stating and proving this theorem. $G(x)$ is defined by (11) and its Mellin transform $M(s)$ by (12). We write

$$
M(s)=\frac{N(s)}{D(s)}
$$

where

$$
N(s)=\prod_{m=1}^{q} \Gamma\left(b_{m}+c s\right) \prod_{n=1}^{p} \Gamma\left(1-a_{n}-c s\right) .
$$

From (12) it is evident that

$$
N(1-s)=D(s) \text {. }
$$

THEOREM 4. If (i) condition (i) of Theorem 1 holds, (ii) $E(1 / 2-s)$ is an even function of $s$, (iii) $N(s) E(s) \in L_{2}(1 / 2-i \infty, 1 / 2+i \infty)$,

$$
\text { (iv) } f(x)=\frac{1}{2 \pi i} \int_{1 / 2-i \infty}^{1 / 2+i \infty} N(s) E(s) x^{-s} d s \text {, }
$$

then

$$
\int_{0}^{x} f(x) d x=\int_{0}^{\infty} f(y) \frac{G_{1}(x y)}{y} d y .
$$

If we can differentiate with respect to $x$ through the integral sign then (46) reduces to (38) and $f(x)$, as defined by (45), is then a self reciprocal function for $G(x)$. Whether we can differentiate through the integral sign of (46) or not we shall say that $f(x)$ is a self reciprocal for $G(x)$ if $(46)$ is satisfied. The advantage of this definition over (38) is that we can prove (46) by using the much easier theory of convergence in mean square whereas the proof of (38) requires the use of ordinary convergence methods.

Theorem 4 is proved by means of two applications of the Parseval theorem, Theorem $72[6$, p. 95]. From $(17)$ it follows that $M(s) /(1-s)$ $\in L_{2}(1 / 2-i \infty, 1 / 2+i \infty)$ and that $G_{1}(x) / x$ is its Mellin transform. Hence, using $y$ as the Mellin transform variable, it follows that $G_{1}(x y) / y$ and $M(s) x^{1-s} /(1-s)$ are Mellin transforms of each other. From condition (iii) and Theorem $72[6$, p. 95] we can apply the Parseval theorem and obtain

$$
\begin{aligned}
\int_{0}^{\infty} f(y) \frac{G_{1}(x y)}{y} d y & =\frac{1}{2 \pi i} \int_{1 / 2-i \infty}^{1 / 2+i \infty} \frac{M(s) x^{1-s}}{(1-s)} N(1-s) E(1-s) d s \\
& =\frac{1}{2 \pi i} \int_{1 / 2-i \infty}^{1 / 2+i \infty} N(s) E(s) \frac{x^{1 \rightarrow s}}{(1-s)} d s,
\end{aligned}
$$


on using (42), (44) and condition (ii).

Again, using $y$ as the Mellin transform variable, the function $F(y)=1$, $y<x$ and $F(y)=0, y>x$, has $x^{s} / s$ for its Mellin transform. Since $F(y)$ $\in L_{2}(0, \infty)$ we may apply the Parseval theorem once again (Theorem 72 $[6$, p. 95]). In (45), which defines $f(x)$, replace $x$ by $y$ and use the $F(y)$ just defined; we then have

$$
\begin{aligned}
\int_{0}^{x} f(y) d y & =\int_{0}^{\infty} f(y) F(y) d y \\
& =\frac{1}{2 \pi i} \int_{1 / 2-i \infty}^{1 / 2+i \infty} N(s) E(s) \frac{x^{1-s}}{(1-s)} d s .
\end{aligned}
$$

Comparison of (48) and (50) evidently completes the proof of Theorem 4.

Theorem 4 contains as special cases such well known self reciprocal functions as $\exp \left(-x^{2} / 2\right)$ for $\cos x$ and $x \exp \left(-x^{2} / 2\right)$ for $\sin x$.

6. The $H(x)$ function. A more general function than the $G$-function of (5) is the function $f(x)$ defined by the following equation:

$$
f(x)=\frac{1}{2 \pi i} \int_{T} \frac{\prod_{i=1}^{m} \Gamma\left(b_{i}+c_{i} s\right) \prod_{i=1}^{n} \Gamma\left(a_{i}-e_{i} s\right)}{\prod_{i=m+1}^{q} \Gamma\left(b_{i}-c_{i} s\right) \prod_{i=n+1}^{p} \Gamma\left(a_{i}+e_{i} s\right)} x^{-s} d s
$$

This reduces to the $G$-function of (5) when $c_{i}=c, i=1, \cdots, q, e_{i}=c$, $i=1, \cdots, p, a_{i}$ is replaced by $1-a_{i}, i=1, \cdots, n$ and $b_{i}$ is replaced by $1-b_{i}, i=m+1, \cdots, q$. As far as I am aware these functions have not so far been studied to any great extent but they possess one advantage in common with the $G$-function. For a suitable contour $T$ the Mellin transform of $f(x)$ is evidently the coefficient of $x^{-s}$ in the integrand of (51). Consequently the question whether the Mellin transform of $f(x)$ does or does not satisfy the functional equation is easily answered and this answer helps us to decide whether $f(x)$ can or cannot be a symmetrical Fourier kernel.

We now restrict $f(x)$ to $H(x)$ whose Mellin transform does satisfy (6). $H(x)$ is defined by

$$
H(x)=\frac{1}{2 \pi i} \int_{T} \frac{\prod_{m=1}^{q} \Gamma\left(b_{m}+c_{m} s\right) \prod_{n=1}^{p} \Gamma\left(a_{n}-e_{n} s\right)}{\prod_{m=1}^{q} \Gamma\left(b_{m}+c_{m}-c_{m} s\right) \prod_{n=1}^{p} \Gamma\left(a_{n}-e_{n}+e_{n} s\right)} x^{-s} d s
$$

where the following simplifying assumptions are made:

(i) $c_{m}>0, m=1, \cdots, q ; e_{n}>0, n=1, \cdots, p$,

(ii) all the poles of the integrand of (52) are simple, 
(iii) the contour $T$ is a straight line parallel to the imaginary axis in the $s(=\sigma+i t)$ plane and the poles of $\Gamma\left(b_{m}+c_{m} s\right)$ lie on the left of $T$ while those of $\Gamma\left(a_{n}-e_{n} s\right)$ lie on the right of $T$.

$$
\text { (iv) } \quad D=2\left(\sum_{m=1}^{q} c_{m}-\sum_{n=1}^{p} e_{n}\right)>0 \text {. }
$$

The quantity $D$ plays in the $H(x)$ theory a part analogous to that played by the quantity $h$ in the $G(x)$ theory in $\$ 2$.

On writing (52) in the form

$$
H(x)=\frac{1}{2 \pi i} \int_{T} P(s) x^{-s} d s
$$

it is evident that

$$
P(s) P(1-s)=1 \text {. }
$$

We now use the asymptotic expansion of the Gamma function (14) to determine the order of $P(s), s=\sigma+i t, \sigma$ and $t$ real, when $t$ is large and either positive or negative. The result is

$$
P(s)=|t|^{D(\sigma-1 / 2)} \exp \{i t(D \log t-\log x-D)\}\left\{Q+O\left(|t|^{-1}\right)\right\},
$$

where $Q$ is a constant which may have one value when $t$ is large and positive and another value when $t$ is large and negative. From (56) we see that if $\sigma<1 / 2$ then the integral of (54) is uniformly convergent with respect to $x$. We may integrate through the integral sign of (54) and therefore if

$$
H_{1}(x)=\int_{0}^{x} H(x) d x
$$

then

$$
H_{1}(x)=\frac{1}{2 \pi i} \int_{T} \frac{P(s)}{(1-s)} x^{1-s} d s .
$$

This has been proved only when $\sigma<1 / 2$ but in the next two theorems the contour $T$ will be taken along the line $\sigma=1 / 2$ and so it is necessary to extend the proof. To do this we note that on a large circle whose points have polar co-ordinates $(R, \theta)$ the dominant factor in $P(s)$ is

$$
\exp \{D(R \log R) \cos \theta\} \text {. }
$$

Since $D>0$ we may close the contour $T$ of (54) by a large semicircle on the left and express $H(x)$ in terms of the residues of $\Gamma\left(b_{m}+c_{m} s\right), m=1, \cdots, q$. For $H(x)$ we then obtain $q$ power series, each of which is an entire function.

For the integral of (58), on multiplying (56) by $1 / t$ it follows that the 
integral converges when $\sigma=1 / 2$. Again (59) is the dominant factor for the integrand of (58) on a large circle so that the contour $T$ can be closed by a large semicircle on the left. The integral can then be computed by means of the poles of $\Gamma\left(b_{m}+c_{m} s\right)$. We then find easily that the residues are the terms of $H(x)$ integrated with respect to $x$. Since the power series expressing $H(x)$ are all entire functions such term by term integration is permissible and so (57) and (58) are still true when $\sigma=1 / 2$.

From (56) $P(s)$ is bounded on the line $\sigma=1 / 2$ and therefore $P(s) /(1-s)$ $\in L_{2}(1 / 2-i \infty, 1 / 2+i \infty)$. Consequently we may evaluate the integral of (58) either by the methods of ordinary convergence or by the methods of convergence in mean square and both these evaluations must be the same, except for a set of points of zero measure. Consequently if the integral of (58) is evaluated by the methods of convergence in mean square the result will be $H_{1}(x)$ as defined by (57). We can now proceed with the statement and proof of the next two theorems.

\section{Convergence in mean square theorems.}

TheOREM 5. $\operatorname{Re}(z)$ denotes the real part of $z$. If (i) $c_{m}>0, m=1, \cdots, q$, $e_{n}>0, n=1, \cdots, p$ and

$$
D=2\left(\sum_{m=1}^{q} c_{m}-\sum_{n=1}^{p} e_{n}\right)>0,
$$

(ii) $\operatorname{Re}\left(b_{m}\right)>-c_{m} / 2, m=1, \cdots, q$, $\operatorname{Re}\left(a_{n}\right)>e_{n} / 2, n=1, \cdots, p$,

(iii) $f(x) \in L_{2}(0, \infty)$ then

$$
g(x)=\frac{d}{d x} \int_{0}^{\infty} H_{1}(x u) f(u) \frac{d u}{u}
$$

defines, almost everywhere, a function $g(x) \in L_{2}(0, \infty)$,

$$
f(x)=\frac{d}{d x} \int_{0}^{\infty} H_{1}(x u) g(u) \frac{d u}{u}
$$

holds almost everywhere and

$$
\int_{0}^{\infty}\{f(x)\}^{2} d x=\int_{0}^{\infty}\{g(x)\}^{2} d x .
$$

Before starting on the proof we note that the inequality for $\operatorname{Re}\left(a_{n}\right)$ above differs from that of Theorem 1. This is due to the fact that in the definition of $G(x)$ we have used $1-a_{n}$ whereas in the definition of $H(x)$ we have replaced $1-a_{n}$ by $a_{n}$.

As in the case of Theorem 1 the theorem above is proved by an appeal to Theorem 129 [6, p. 222]. 
First we observe that conditions (i) and (ii) above ensure that the poles of $\Gamma\left(b_{m}+c_{m} s\right), m=1, \cdots, q$, lie on the left of the line $\sigma=1 / 2(s=\sigma+i t)$ and that the poles of $\Gamma\left(a_{n}-e_{n} s\right)$ lie on the right of $\sigma=1 / 2$. Hence we can choose the line $\sigma=1 / 2$ for the contour $T$ in (58).

We now proceed to establish the truth of the three conditions of Theorem 129 [6, p. 222]. They are (i) that $P(1 / 2+i t) P(1 / 2-i t)=1$, which follows from (55), (ii) that $H_{1}(x)$ and $P(s)$ should be related as in (58) and (iii) that $f(x) \in L_{2}(0, \infty)$ which is covered by condition (iii) above. Since the conditions of Theorem 129 [6, p. 222] are satisfied the conclusions of that theorem must also be true and these conclusions are (a), (b) and (c) above. This completes the proof of Theorem 5 .

We now proceed to obtain a formula for the self reciprocal functions of $H(x)$. The following results are useful in the statement of the theorem. We write

$$
P(s)=\frac{Q(s)}{W(s)}
$$

where

$$
Q(s)=\prod_{m=1}^{q} \Gamma\left(b_{m}+c_{m} s\right) \prod_{n=1}^{p} \Gamma\left(a_{n}-e_{n} s\right) .
$$

Here $P(s)$ is the coefficient of $x^{-s}$ in the integral of (52) and so

$$
Q(1-s)=W(s) \text {. }
$$

We can now state the theorem in question.

THEOREM 6. If (i) conditions (i) and (ii) of Theorem 5 both hold, (ii) $E(1 / 2-s$ ) is an even function of $s$, (iii) $Q(s) E(s) \in L_{2}(1 / 2-i \infty, 1 / 2+i \infty)$,

$$
\text { (iv) } f(x)=\frac{1}{2 \pi i} \int_{1 / 2-i \infty}^{1 / 2+i \infty} Q(s) \cdot E(s) x^{-s} d s
$$

then

$$
\int_{0}^{x} f(x) d x=\int_{0}^{\infty} f(y) \frac{H_{1}(x y)}{y} d y .
$$

If we can differentiate with respect to $x$ through the integral sign on the right of (63) we obtain a formula akin to (38) and $f(x)$ is then a self reciprocal function for the kernel $H(x)$. But even if this differentiation cannot be performed we shall still say that (63) defines $f(x)$ as a self reciprocal function for $H(x)$.

The proof of Theorem 6 above is almost identical with that of Theorem 4, $\S 6$. The Parseval theorem for the Mellin transform, Theorem $72[6$, p. 95] is applied twice along the lines of equations (48) and (50) of $\$ 6$. Further details 
are hardly necessary and we shall therefore continue with the next theorem.

8. The case of ordinary convergence. As frequently happens in Fourier transform theory, theorems using the methods of ordinary convergence appear to be much more difficult to establish than those which use the methods of convergence in mean square.

THEOREM 7. $\operatorname{Re}(z)$ denotes the real part of $z$. If (i) $c_{m}>0, m=1, \cdots, q$; $e_{n}>0, n=1, \cdots, p ;$ and

$$
D=2\left(\sum_{m=1}^{q} c_{m}-\sum_{n=1}^{p} e_{n}\right)>0,
$$

(ii) $\operatorname{Re}\left(b_{m}\right) \geqq c_{m}(1-D) / 2 D, m=1, \cdots, q$, $\operatorname{Re}\left(a_{n}\right)>e_{n}(1+D) / 2 D, n=1, \cdots, p$

(iii) $f(y) y^{(1-D) / 2 D} \in L(0, \infty)$ and $f(y)$ is of bounded variation near $y=x$, $(x>0)$ then

$$
\int_{0}^{\infty} H(x u)\left\{\int_{0}^{\infty} H(u y) f(y) d y\right\} d u=\frac{1}{2}\{f(x+0)+f(x-0)\} .
$$

Once again the inequality for $\operatorname{Re}\left(a_{n}\right)$ above differs from that of $\operatorname{Re}\left(a_{n}\right)$ in condition (ii) Theorem 2. This is so because $1-a_{n}$ is used in Theorem 2 while $a_{n}$ is used instead in Theorem 7 .

As in the case of Theorem 2 we prove Theorem 7 by first transforming (64) into a form to which Theorem 134 [6, p. 232] can be applied. For future use we define a quantity $\alpha$ as follows:

$$
\alpha=\prod_{m=1}^{q}\left(c_{m} / D\right)^{2 c_{m} / D} \prod_{n=1}^{p}\left(e_{n} / D\right) e^{-2 e_{n} / D} .
$$

Since all the terms on the right hand side of (65) are positive it is evident that (65) defines $\alpha$ as a unique positive quantity. We now write

$$
x=X^{D}, \quad y=Y^{D} \text { and } u=(U \alpha)^{D}
$$

in (64). We then obtain, on allowing for $d y=D Y^{D-1} d Y$ and $d u=D U^{D-1} \alpha^{D} d U$,

$$
\begin{aligned}
\int_{0}^{\infty} H & \left\{(X U \alpha)^{D}\right\}(X U \alpha)^{(D-1) / 2} D \alpha^{1 / 2} \\
\cdot & {\left[\int_{0}^{\infty} H\left\{(U Y \alpha)^{D}\right\}(U Y \alpha)^{(D-1) / 2} D \alpha^{1 / 2} f\left(Y^{D}\right)(Y / X)^{(D-1) / 2} d Y\right] d U } \\
& =\frac{1}{2}\left\{f\left(X^{D}+0\right)+f\left(X^{D}-0\right)\right\} .
\end{aligned}
$$

We aim at proving (64) by proving (67) first. Evidently the kernel of (67) is $H\left\{(X \alpha)^{D}\right\}(X \alpha)^{(D-1) / 2} D \alpha^{1 / 2}$ and from (52) we have 


$$
\begin{aligned}
& H\left\{(X \alpha)^{D}\right\}(X \alpha)^{(D-1) / 2} D \alpha^{1 / 2} \\
& =\frac{1}{2 \pi i} \int \frac{\prod_{m=1}^{q} \Gamma\left(b_{m}+c_{m} s\right) \prod_{n=1}^{p} \Gamma\left(a_{n}-e_{n} s\right)}{\prod_{m=1}^{q} \Gamma\left(b_{m}+c_{m}-c_{m} s\right) \prod_{n=1}^{p} \Gamma\left(a_{n}-e_{n}+e_{n} s\right)}(X \alpha)^{(-2 D s+D-1) / 2} D \alpha^{1 / 2} d s .
\end{aligned}
$$

We now make one more transformation

$$
(-2 D s+D-1) / 2=-S .
$$

Equation (68) then becomes

$$
H\left\{(X \alpha)^{D}\right\}(X \alpha)^{(D-1) / 2} D \alpha^{1 / 2}=\frac{1}{2 \pi i} \int Z(s) X^{-S} d S,
$$

where

$$
Z(S)=\frac{\prod_{m=1}^{q} \Gamma\left(b_{m}+\frac{c_{m}(D-1)}{2 D}+\frac{c_{m} S}{D}\right) \prod_{n=1}^{p} \Gamma\left(a_{n}-\frac{e_{n}(D-1)}{2 D}-\frac{e_{n} S}{D}\right)}{\prod_{m=1}^{q} \Gamma\left(b_{m}+c_{m}-\frac{c_{m}(D-1)}{2 D}-\frac{c_{m} S}{D}\right) \prod_{n=1}^{p} \Gamma\left(a_{n}-e_{n}+\frac{e_{n}(D-1)}{2 D}+\frac{e_{n} S}{D}\right)} \alpha^{1 / 2-S}
$$

where the contour $T$ is any straight line parallel to the imaginary axis in the $S(=\sigma+i t)$ plane for which $0<\sigma<1 / 2$. The corresponding contour for (68) is easily deduced from this statement by means of the transformation (69).

As a consequence of these transformations we can now proceed to prove (64) by first proving (67) and (67) is proved in turn by using Theorem 134 $[6$, p. 232]. The last theorem is based upon six conditions of which four apply to $Z(S)$, the Mellin transform of the kernel in (67) and of the function on the left of (70), and two to $f\left(Y^{D}\right)$. We now show that all these six conditions are satisfied.

With $S=\sigma+i t, \sigma$ and $t$ real, the first condition is that we can find $\sigma_{0}<0$ and $\sigma_{1}>1$ such that $Z(S)$ is regular in the strip $\sigma_{0}<\sigma<\sigma_{1}$, except possibly for a finite number of simple poles on the imaginary axis $\sigma=0$. The poles of (71) are evidently at

$$
-\frac{D}{c_{m}}\left(r+b_{m}+\frac{c_{m}(D-1)}{2 D}\right) \text { and } \frac{D}{e_{n}}\left(r+a_{n}-\frac{e_{n}(D-1)}{2 D}\right),
$$

$r=0,1,2, \cdots$. From condition (ii) in the statement of Theorem 7 it follows that all the $a_{n}$ poles lie on the right of $\sigma=1$ and that if, in the case of $b_{m}$, only the inequality holds then all the $b_{m}$ poles lie on the left of $\sigma=0$. If $\operatorname{Re}\left(b_{m}\right)$ $=c_{m}(1-D) / 2 D$ for some values of $m$ then we have poles on the imaginary axis. But there are at most $q$ of such poles on $\sigma=0$. Since the singularities of $Z(S)$ are all isolated simple poles it follows that $\sigma_{0}<0$ and $\sigma_{1}>1$ can be found such that $Z(S)$ is regular inside the strip $\sigma_{0}<\sigma<\sigma_{1}$ except possibly for some simple poles on the imaginary axis. 
The second condition is the most difficult of the six. We must establish that for large positive or negative $t, S=\sigma+i t$,

$$
Z(S)=\Gamma(S) \cos (S \pi / 2)\left\{\beta+\frac{\gamma}{S}+O\left(|S|^{-2}\right)\right\},
$$

where $\beta$ and $\gamma$ are constants which may have one value when $t$ is large and positive and another value when $t$ is large and negative. $O$ is the usual order symbol.

To establish this we must examine the asymptotic expansion of $Z(S)$ in some detail. Consider the following factors of $Z(S)$,

$$
\frac{\Gamma\left(b_{m}+c_{m}(D-1) / 2 D+c_{m} S / D\right)}{\Gamma\left(b_{m}+c_{m}-c_{m}(D-1) / 2 D-c_{m} S / D\right)} .
$$

On using $\Gamma(z) \Gamma(1-z)=\pi / \sin (\pi z),[7$, p. 233], we may replace the Gamma function in the denominator of (74) by a Gamma function containing $+c_{m} S / D$ instead of $-c_{m} S / D$. We then apply to (74) the asymptotic expansion of the Gamma function, (14), for large $|S|$ and $|\arg S|<\pi-\delta, \delta>0$. After some simplification of the exponential terms it then follows that the asymptotic expansion of (74) is the product of the following two factors, (75a) and (75b),

$$
\exp \left[\frac{2 c_{m}}{D}\left\{\left(S-\frac{1}{2}\right) \log S-S\right\}+\left\{\frac{2 c_{m}}{D}\left(S-\frac{1}{2}\right) \log \left(\frac{c_{m}}{D}\right)\right\}\right]
$$

and

$$
\left[\sin \pi\left\{b_{m}+c_{m}-\frac{c_{m}(D-1)}{2 D}-\frac{c_{m} S}{D}\right\}\right]\left[\beta_{m}+\gamma_{m} S^{-1}+O\left(|S|^{-2}\right)\right]
$$

where $\beta_{m}$ and $\gamma_{m}$ are constants. (75a) and (75b) will appear in the numerator of $Z(S)$ but the corresponding factors for the Gamma functions involving $a_{n}$ and $e_{n}$ will appear in the denominator of $Z(S)$, since the Gamma function containing $+e_{n} S / D$ is now in the denominator.

Among the factors in the asymptotic expansion of $Z(S)$ we have

$$
\begin{array}{r}
\prod_{m=1}^{q} \exp \left[\frac{2 c_{m}}{D}\left\{\left(S-\frac{1}{2}\right) \log S-S\right\}\right] \\
\prod_{n=1}^{p} \exp \left[\frac{2 e_{n}}{D}\left\{\left(S-\frac{1}{2}\right) \log S-S\right\}\right] \\
=\exp \left\{\left(S-\frac{1}{2}\right) \log S-S\right\} \\
=\Gamma(S)\left\{\lambda+\mu S^{-1}+O\left(|S|^{-2}\right)\right\}
\end{array}
$$


where $\lambda$ and $\mu$ are constants. (77) follows from (76) because of the definition of $D$ in condition (i) of Theorem 7 and (78) follows from (77) because of the asymptotic expansion of $\Gamma(S)$. (78) accounts only for part of the factors which arise from (74). The remaining part gives us, as a factor in the asymptotic expansion of $Z(S)$,

$$
\frac{\prod_{m=1}^{q} \exp \left\{\frac{2 c_{m}}{D}\left(S-\frac{1}{2}\right) \log \left(\frac{c_{m}}{D}\right)\right\}}{\prod_{n=1}^{p} \exp \left\{\frac{2 e_{n}}{D}\left(S-\frac{1}{2}\right) \log \left(\frac{e_{n}}{D}\right)\right\}}=\alpha^{S-1 / 2}
$$

on using the definition of $\alpha$ in (65). The two expressions (78) and (79) account for all the factors of the type (75a).

We must now consider the contribution of factors such as (75b) to the asymptotic expansion of $Z(S)$. As in (31) we have

$$
\frac{\sin \pi\left\{b_{m}+\frac{c_{m}(D-1)}{2 D}-\frac{c_{n} S}{D}\right\}}{\left(\cos \frac{1}{2} S \pi\right)^{2 c_{m} / D}}=\phi_{m}+O\left(e^{-|t|}\right),
$$

where the constant $\phi_{m}$ may have one value when $t$ is large and positive and another when $t$ is large and negative. Consequently we have

$$
\frac{\prod_{m=1}^{q} \sin \pi\left\{b_{m}+c_{m}-\frac{c_{m}(D-1)}{2 D}-\frac{c_{m} S}{D}\right\}}{\prod_{n=1}^{p} \sin \pi\left\{a_{n}-\frac{e_{n}(D-1)}{2 D}-\frac{e_{n} S}{D}\right\}}=\cos \left(\frac{1}{2} S \pi\right)\left\{\psi+O\left(e^{-|t|}\right)\right\}
$$

where again the constant $\psi$ depends upon whether $t$ is positive or negative.

We can summarize all these results as follows. The asymptotic expansion of $Z(S), S=\sigma+i t$, for large positive or negative $t$ consists of five factors. They are (i) (78), (ii) (79), (iii) (81), (iv) a group of factors of the type $\beta_{m}+\gamma_{m} S^{-1}$ $+O\left(|S|^{-2}\right)$ which are associated with $(75 \mathrm{~b})$ and therefore should be associated with (81). (iv) has $q$ such factors in the numerator and $p$ in the denominator of $Z(S)$ and their first terms never vanish (in fact all the first terms, such as $\beta_{m}$, are equal to 2 whether in the numerator or in the denominator). Hence all the factors of this group can be amalgamated into one factor of the type

$$
u+v S^{-1}+O\left(S^{-2}\right)
$$

where $u$ and $v$ are constants independent of $t$. The remaining factor $(v)$ is the last term in (71) namely $\alpha^{1 / 2-S}$, which so far has not been included in any of the discussions. 
On multiplying all these five factors together it is evident that (ii) and (v) cancel each other and that the remaining three multiply to the right hand side of (73). This establishes the truth of (73) and proves, therefore, that the second condition of Theorem 134 [6, p. 232] is satisfied.

The remaining four conditions are easily dealt with. The third condition of Theorem $134[6$, p. 232] is that

$$
Z(S) Z(1-S)=1
$$

This is evident from (71).

The fourth condition is that

$$
H\left\{(X \alpha)^{D}\right\}(X \alpha)^{(D-1) / 2} D \alpha^{1 / 2}
$$

must be the Mellin transform of $Z(S)$. This follows from the way in which $Z(S)$ has been defined in (70).

The fifth condition is that

$$
\int_{0}^{\infty}\left|f\left(Y^{D}\right)\right| Y^{(D-1) / 2} d Y
$$

should exist. On writing $Y^{D}=y$ in this integral we see that this condition is covered by the first part of condition (iii) Theorem 7 .

The sixth and last condition is that $f\left(Y^{D}\right) Y^{(D-1) / 2}$ must be of bounded variation near $Y=X, X>0$. This is evidently covered by the last part of condition (iii) Theorem 7.

Since all the six conditions of Theorem $134[6$, p. 232] are satisfied, the conclusions of that theorem must follow and consequently equation (67) is true. By using the transformation (66) in reverse it follows that (64) is true and this completes the proof of Theorem 7 .

9. Discontinuous integrals associated with $H(x)$.

Theorem 8. If (i) $H(x)$ is defined by (52) and $H_{1}(x)$ by (57) and (ii) conditions (i) and (ii) of Theorem 7 hold then

$$
\begin{aligned}
\int_{0}^{\infty} H(x u) \frac{H_{1}(u y)}{u} d u=\frac{1}{2}, & x>y>0, \\
1, & y>x>0 .
\end{aligned}
$$

The proof of Theorem 8 is exactly as for Theorem $3, \S 5$.

10. The asymptotic expansion of $H(x)$ for positive $x$. Since little has been written on the $H$-function it is desirable to determine its asymptotic expansion. A complete investigation for complex as well as real values of $x$ would undoubtedly require a very lengthy discussion as can be seen by considering the writings of Barnes [1] and Meijer [4]. For this reason I confine myself 
to the case of $x$ real and positive. I will use a method which I originated in a previous paper on Fourier kernels [3].

We start with $H(x)$ defined by (52) using, as before, $s=\sigma+i t$, where $\sigma$ and $t$ are real and $\operatorname{Re}(z)$ to denote the real part of $z$. The conditions $\operatorname{Re}\left(b_{m}\right)$ $>0, m=1, \cdots, q$, and $\operatorname{Re}\left(a_{n}\right)>e_{n} / 2, n=1, \cdots, p$, of Theorem 9 below keep the poles of $\Gamma\left(b_{m}+c_{m} s\right), m=1, \cdots, q$, to the left of the line $\sigma=0$ and the poles of $\Gamma\left(a_{n}-e_{n} s\right), n=1, \cdots, p$, to the right of the line $\sigma=1 / 2$. Hence we may use the line $\sigma=\mu$ for the contour of (52) if $0<\mu<1 / 2$, and the integral of (52) will then converge if (and only if) $x$ is real and positive. It is for this reason that our method must be restricted to real values of $x$, but against this we can offset the fact that our method is a simple and a comparatively quick one.

In addition to the quantities $D$ of (53) and $\alpha$ of (65) we need two further constants, $\beta$ and $K$, defined by (86) below

$$
\beta=\alpha^{D}, \quad K=2\left\{\sum_{m=1}^{q}\left(b_{m}+c_{m}\right)-\sum_{n=1}^{p} a_{n}\right\} .
$$

We are now given $\sigma_{0}$ and our problem is to find an asymptotic expansion for $H(x)$, when $x$ is large and positive, with an error term $O\left(x^{-\sigma_{0}}\right)$, where $O$ is the usual order symbol.

Theorem 9. Assumptions: (i) $c_{m}>0, m=1, \cdots, q, e_{n}>0, n=1, \cdots, p$, $D>0$ ( $D$ defined in (53)),

(ii) $x$ real and positive, $\operatorname{Re}\left(b_{m}\right)>0, m=1, \cdots, q, \operatorname{Re}\left(a_{n}\right)>e_{n} / 2, n$ $=1, \cdots, p$,

(iii) given $\sigma_{0}>1 / 2, r$ denotes the greatest integer in $\left\{D\left(2 \sigma_{0}-1\right)+3\right\} / 2$ and $u_{n}$ denotes the greatest positive integer in $e_{n} \sigma_{0}-\operatorname{Re}\left(a_{n}\right), n=1, \cdots, p$,

(iv) $q-p$ is an odd positive integer.

Conclusion:

$$
\begin{aligned}
& H(x)=\left(\frac{x}{\beta}\right)^{(1-D) / 2 D} \sum_{i=0}^{r} \nu_{i}\left(\frac{x}{\beta}\right)^{-i / D} \sin \left\{\frac{1}{2} \pi\left(K-i+\frac{1-D}{2}\right)+\left(\frac{x}{\beta}\right)^{1 / D}\right\} \\
& +\sum_{n=1}^{p}\left\{x^{-a_{n} / e_{n}}\left(A_{n}+B_{n} x^{-1 / e_{n}}+C_{n} x^{-2 / e_{n}}+\cdots+U_{n} x^{-u_{n} / e_{n}}\right)\right\} \\
& +O\left(x^{-\sigma_{0}}\right),
\end{aligned}
$$

where $\nu_{0}, \nu_{1}, \cdots, \nu_{r}$ and $A_{n}, B_{n}, \cdots, U_{n}$ are constants which depend upon $b_{m}$ and $c_{m}, m=1, \cdots, q$ and $a_{n}$ and $e_{n}, n=1, \cdots, p$ but are independent of $x$. The constants $\nu_{0}, \nu_{1}, \cdots, \nu_{r}$ are computed by a method specified in Lemma 6, $\$ 11$ and the constants $A_{n}, B_{n}, \cdots, U_{n}, n=1, \cdots, p$, are computed by means of residues as specified in $\$ 12$. (53) gives $D$ and (86) $\beta$ and $K$.

If $q-p$ is an even positive integer (instead of condition (iv) above) then in the right hand side of (87) we must replace 


$$
\sin \left\{\frac{1}{2} \pi\left(K-i+\frac{1-D}{2}\right)+\left(\frac{x}{\beta}\right)^{1 / D}\right\}
$$

by

$$
\cos \left\{\frac{1}{2} \pi\left(K-i+\frac{1-D}{2}\right)+\left(\frac{x}{\beta}\right)^{1 / D}\right\} .
$$

It is possible to merge these two cases, $q-p$ odd or even, into one, but the formulae become more complex. Also I think that it is desirable to show this difference between the two cases clearly.

It is possible to exhibit the difference between these two cases in another manner. The term $i \pi / 2(i=0,1, \cdots, r)$ in each of the trigonometrical functions of (87) shows that in the trigonometrical sum the sines and cosines appear alternately beginning with a sine term when $(q-p)$ is odd and a cosine term when $(q-p)$ is even.

The proof of Theorem 9 depends upon some easily proved lemmas which are dealt with in the next section.

11. Some preliminary lemmas. Conditions (ii) of Theorem 9 enable us to use (52) as the definition of $H(x)$ with the line $\sigma=\sigma_{0}$ where $0<\sigma_{0}<1 / 2$. We now write (52) in the form

$$
H(x)=\frac{1}{2 \pi i} \int Q(s)\left(\frac{x}{\beta}\right)^{-s} d s,
$$

using the contour $\sigma=\sigma_{0}$ just mentioned and with

$$
Q(s)=\frac{\prod_{m=1}^{q} \Gamma\left(b_{m}+c_{m} s\right) \prod_{n=1}^{p} \Gamma\left(a_{n}-e_{n} s\right)}{\prod_{m=1}^{q} \Gamma\left(b_{m}+c_{m}+c_{m} s\right) \prod_{n=1}^{p} \Gamma\left(a_{n}-e_{n}+e_{n} s\right)}(\alpha)^{-s D},
$$

since $\beta=\alpha^{D}$.

Lemma 1. The asymptotic expansion of the gamma function [7, p. 272]. For large $|s|,|\arg s|<\pi-\delta, \delta>0$ we have

$$
\Gamma(s+a)=F \cdot \exp \left\{\left(s+a-\frac{1}{2}\right) \log s-s\right\}
$$

where

$$
F=A+B s^{-1}+C s^{-2}+\cdots+R s^{-r}+O\left(|s|^{-(2 r+1) / 2}\right),
$$

the constants $A, B, C, \cdots, R$ being all independent of $s$. It is important to note that $A \neq 0$, in this case $A=(2 \pi)^{1 / 2}$.

Any expression such as $F$ of (93), starting with a constant not equal to 
zero and continuing with negative powers of $s$ until an algebraical order term is reached, will be referred to as a function of type $F$.

Lemma 2. The first amalgamation lemma. A group of functions of type $F$ which are combined by multiplication and division can be amalgamated into one function of type $F$.

The lemma is obvious for the case of multiplication. It is also obvious in the case of division since the constant term of the $F$ type function in the denominator cannot vanish.

Lemma 3. The second amalgamation lemma. Let $s=\sigma+i t$. Then for fixed $\sigma$ and large positive or negative $t$

$$
\left\{\begin{array}{c}
\prod_{m=1}^{q} \sin \pi\left(b_{m}+c_{m}-c_{m} s\right) \\
\prod_{n=1}^{p} \sin \pi\left(a_{n}-e_{n} s\right)
\end{array}\right\}=\begin{aligned}
& \left\{\sin \frac{1}{2} \pi(K-D s)\right\}\left\{\lambda_{1}+O\left(e^{-2|t|}\right)\right\} \\
& \left\{\cos \frac{1}{2} \pi(K-D s)\right\}\left\{\lambda_{2}+O\left(e^{-2|t|}\right)\right\}
\end{aligned}
$$

where the upper expression on the right hand side of (94) is to be used when $(q-p)$ is an odd positive integer and the lower expression when $(q-p)$ is an even positive integer. Also $\lambda_{1}=(2 i)^{(p-q+1)}$ and $\lambda_{2}=2(2 i)^{(p-q)}$, both being real.

To prove this we write $s=\sigma+i t$ and note that for large positive $t$ we have

$$
\sin \pi\left(b_{m}+c_{m}-c_{m} s\right)=\frac{1}{2 i}\left[\exp \left\{i \pi\left(b_{m}+c_{m}-c_{m} s\right)\right\}\right]\left[1-O\left(e^{-2 t}\right)\right]
$$

and that for large negative $t$ we have

$$
\sin \pi\left(b_{m}+c_{m}-c_{m} s\right)=-\frac{1}{2 i}\left[\exp \left\{-i \pi\left(b_{m}+c_{m}-c_{m} s\right)\right\}\right]\left[1-O\left(e^{-2|t|}\right)\right] .
$$

There are also corresponding expressions for $\sin \pi\left(a_{n}-e_{n} s\right)$.

These values are now inserted in the left hand side of (94) and the result simplified by using $D$ of (53) and $K$ of (86). It is then clear that the left hand side behaves like

$$
\frac{\lambda_{1}}{2 i}\left[\exp \left\{\frac{1}{2} i \pi(K-D s)\right\}\right]\left[1-O\left(e^{-2|t|}\right)\right]
$$

for large positive values of $t$ and like

$$
(-1)^{(q-p)} \frac{\lambda_{1}}{2 i}\left[\exp \left\{-\frac{1}{2} i \pi(K-D s)\right\}\right]\left[1-O\left(e^{-2|t|}\right)\right]
$$

for large negative values of $t$.

Finally, whether $t$ is large and positive or large and negative, these expressions in turn behave like 


$$
\left\{\sin \frac{1}{2} \pi(K-D s)\right\}\left\{\lambda_{1}+O\left(e^{-2|t|}\right)\right\}
$$

when $(q-p)$ is odd and like

$$
\left\{\cos \frac{1}{2} \pi(K-D s)\right\}\left\{\lambda_{2}+O\left(e^{-2|\iota|}\right)\right\}
$$

when $(q-p)$ is even. This completes the proof of the lemma.

Lemma 4. For large $|s|,|\arg s|<\pi-\delta, \delta>0$,

$$
\begin{aligned}
\frac{\Gamma\left(b_{m}+c_{m} s\right)}{\Gamma\left(b_{m}+c_{m}-c_{m} s\right)}=\exp [ & \left.2 c_{m}\left\{\left(s-\frac{1}{2}\right) \log s-s\right\}\right] \\
& \times \exp \left\{\left(s-\frac{1}{2}\right) 2 c_{m} \log c_{m}\right\} \\
& \times\left\{\sin \pi\left(b_{m}+c_{m}-c_{m} s\right)\right\} F
\end{aligned}
$$

where $F$ is a function of type $F$ defined in Lemma 1.

To prove this we first write

$$
\frac{1}{\Gamma\left(b_{m}+c_{m}-c_{m} s\right)}=\Gamma\left(1-b_{m}-c_{m}+c_{m} s\right) \pi \sin \pi\left(b_{m}+c_{m}-c_{m} s\right),
$$

[7, p. 233] in (95) and then use the asymptotic expansion of Lemma 1. The sine and $F$ terms on the right of (95) are obvious so that we need only discuss the exponential terms. After absorbing the factor $\pi$ in the $F$ term the exponential terms are

$$
\begin{aligned}
& {\left[\exp \left\{\left(b_{m}+c_{m} s-\frac{1}{2}\right) \log c_{m} s-c_{m} s\right\}\right]} \\
& \times\left[\exp \left\{\left(1-b_{m}-c_{m}+c_{m} s-\frac{1}{2}\right) \log c_{m} s-c_{m} s\right\}\right]
\end{aligned}
$$

Evidently the $b_{m}$ terms cancel and after some rearrangements this becomes equivalent to the right hand side of (95), in so far as the exponential terms are concerned.

We also have the analogous formula

$$
\begin{aligned}
\frac{\Gamma\left(a_{n}-e_{n}+e_{n} s\right)}{\Gamma\left(a_{n}-e_{n} s\right)}= & \exp \left[2 e_{n}\{(s-1 / 2) \log s-s\}\right] \\
& \times \exp \left\{(s-1 / 2) 2 e_{n} \log e_{n}\right\} \\
& \times\left\{\sin \pi\left(a_{n}-e_{n} s\right)\right\} F .
\end{aligned}
$$


After inversion (96) can be inserted in the right hand side of (91) in order to obtain estimates for $Q(s)$.

Lemma 5. The Asymptotic eXPANSION FOR $Q(s)$ OF (91). If $s=\sigma+i t, \sigma$ is fixed and $t$ is large and either positive or negative then

$$
Q(s)=\begin{array}{lc}
s^{(1-D) / 2} \Gamma(D s)\{\sin \pi(K-D s) / 2\} F ; & (q-p) \text { odd }, \\
s^{(1-D) / 2} \Gamma(D s)\{\cos \pi(K-D s) / 2\} F ; & (q-p) \text { even, }
\end{array}
$$

where $F$ is a function of type $F$ defined in Lemma 1.

To prove this we must apply (95) and (96) to (91). From Lemma 2 the $F$ terms amalgamate into a single $F$ term and from Lemma 3 the sine terms amalgamate to

$$
\left\{\sin \frac{1}{2} \pi(K-D s)\right\}\left\{\lambda_{1}+O\left(e^{-2|t|}\right)\right\} \text { when }(q-p) \text { is odd }
$$

and to

$$
\left\{\cos \frac{1}{2} \pi(K-D s)\right\}\left\{\lambda_{2}+O\left(e^{-2|t|}\right)\right\} \text { when }(q-p) \text { is even. }
$$

Evidently if $F$ is of type $F$, Lemma $1, \sigma$ is finite and $t$ is large, either positive or negative, then $\left\{\lambda_{1}+O\left(e^{-2|\ell|}\right)\right\} F$ amalgamates into a function of type $F$. Consequently the sine and $F$ factors of (95) and (96) amalgamate to

$$
\left\{\sin \frac{1}{2} \pi(K-D s)\right\} F \quad \text { when } \quad(q-p) \text { is odd }
$$

and to

$$
\left\{\cos \frac{1}{2} \pi(K-D s)\right\} F \text { when }(q-p) \text { is even, }
$$

where $F$ is a function of type $F$ defined in Lemma 1 . This leaves us with the exponential terms of (95) and (96) to deal with together with the factor $(\alpha)^{-s D}$, which occurs in (91). Denote these factors by $E$, then

$$
E=\frac{\prod_{m=1}^{q} \exp \left[2 c_{m}\{(s-1 / 2) \log s-s\}+(s-1 / 2) 2 c_{m} \log c_{m}\right]}{\prod_{n=1}^{p} \exp \left[2 e_{n}\{(s-1 / 2) \log s-s\}+(s-1 / 2) 2 e_{n} \log e_{n}\right]} \alpha^{-s D} .
$$

From (65), which defines $\alpha$, we have 


$$
\frac{\prod_{m=1}^{q} \exp \left\{(s-1 / 2) 2 c_{m} \log c_{m}\right\}}{\prod_{n=1}^{p} \exp \left\{(s-1 / 2) 2 e_{n} \log e_{n}\right\}}=(\alpha D)^{D(2 s-1) / 2}
$$

On using (53), which defines $D$, it then follows that $E$ simplifies to

$$
\begin{aligned}
E & =[\exp D\{(s-1 / 2) \log s-s\}] D^{D(2 s-1) / 2} \alpha^{-D / 2} \\
& =[\exp D\{(s-1 / 2) \log (D s)-s\}] \alpha^{-D / 2} \\
& =[\exp \{(D s-1 / 2) \log (D s)-D s\}](D s)^{(1-D) / 2} \alpha^{-D / 2} \\
& =s^{(1-D) / 2} \Gamma(D s) F^{-1} D^{(1-D) / 2} \alpha^{-D / 2},
\end{aligned}
$$

where we have used the asymptotic expansion of $\Gamma(D s)$ given in Lemma 1 and $F$ denotes a function of type $F$ defined in Lemma 1.

In the last expression we first absorb the constant factors in the function of type $F$ and then associate the result either with (98a) if $(q-p)$ is odd or with $(98 \mathrm{~b})$ if $(q-p)$ is even. This gives us (97) and so completes the proof of this lemma.

LeMma 6. Let $s=\sigma+i t$, where $\sigma$ is fixed and $t$ may be large and either positive or negative. Then we can choose $(r+1)$ constants $\nu_{i}, i=0,1, \cdots, r$, which are independent of $s$ such that

$$
\begin{aligned}
Q(s)-\sum_{i=0}^{r} \nu_{i} \Gamma(D s-i+ & \{1-D\} / 2) \sin \pi(K-D s) / 2 \\
= & O\left(|s|^{(-2 r-1+2 D \sigma-D) / 2}\right), \quad \text { if }(q-p) \text { is odd }
\end{aligned}
$$

or

$$
\begin{aligned}
Q(s)-\sum_{i=0}^{r} \nu_{i} \Gamma(D s-i+ & \{1-D\} / 2) \cos \pi(K-D s) / 2 \\
= & O\left(|s|^{(-2 r-1+2 D \sigma-D) / 2}\right), \quad \text { if }(q-p) \text { is even. }
\end{aligned}
$$

To prove this we first note that from the asymptotic expansion (92)

$$
\begin{aligned}
\Gamma\left(D s-i+\frac{1-D}{2}\right) & =\left[\exp \left\{\left(D s-i+\frac{1-D}{2}-\frac{1}{2}\right) \log D s-D s\right\}\right] F_{i} \\
& =[\exp \{(D s-1 / 2) \log D s-D s\}] s^{(1-D) / 2} s^{-i} F_{i},
\end{aligned}
$$

where $F_{i}$ is a function of type $F$ defined in Lemma 1 . Denote the left hand side of $(100 \mathrm{a})$ by $R(s)$, then from the last result and (97) we have

$$
\begin{gathered}
R(s)=s^{(1-D) / 2}[\exp \{(D s-1 / 2) \log D s-D s\}][\sin \pi(K-D s) / 2] \\
\times\left\{F-\sum_{i=0}^{r} \nu_{i} s^{-i} F_{i}\right\},
\end{gathered}
$$


where in using (97) we have replaced $\Gamma(D s)$ by its asymptotic expansion (92). The terms $F$ and $F_{i}, i=0,1, \cdots, r$, are functions of type $F$ defined in Lemma 1. Each starts with a constant and continues through negative powers of $s$ until an order term is reached. In the case of $F$ the coefficients of the various negative powers of $s$ depend upon $b_{m}, c_{m}, m=1, \cdots, q$ and $a_{n}, e_{n}, n=1, \cdots, p$ only and are independent of $s$. For $F_{i}, i=1, \cdots, r$, these coefficients depend upon $D$, given by (53), and $i$ only. These coefficients are therefore also independent of $s$.

Consider now the expression $\left\{F-\sum_{i=0}^{r} \nu_{i} s^{-i} F_{i}\right\}$. We start by assuming that each of the $F$ type functions terminates with the same order term, namely $O\left(|s|^{-(2 s+1) / 2}\right)$. We can now choose the constants $\nu_{i}, i=0,1, \cdots, r$, successively so that the constant term and the coefficients of $s^{-i}, i=0,2, \cdots, r$, all vanish. Evidently we have $r+1$ equations for the $\nu_{i}, i=0,1, \cdots, r$, constants. It is also evident that these constants $\nu_{i}$, computed in this manner, will depend upon $a_{m}, c_{m}, m=1, \cdots, q, a_{n}, e_{n}, n=1, \cdots, p$, only and will be independent of $s$. The constants can be computed successively since on equating the first term of $\left\{F-\sum_{i=0}^{r} \nu_{i} s^{-i} F_{i}\right\}$ to zero we obtain an equation involving $\nu_{0}$ only, on equating the coefficient of $s^{-1}$ to zero we obtain an equation involving $\nu_{0}$ and $\nu_{1}$ only and so on for higher powers of $s^{-1}$.

This is the method of computation for the constants $\nu_{i}, i=0,1, \cdots, r$, prescribed by the statement of Theorem 9. We shall assume for the rest of this section and also for the next section, $\S 12$, that the constants $\nu_{i}, i=0$, $1, \cdots, r$, are computed in this manner so that we have, for large $s$,

$$
\left\{F-\sum_{i=0}^{r} \nu_{i} s^{-i} F_{i}\right\}=O\left(|s|^{-(2 r+1) / 2}\right) .
$$

We now turn to the other factors of $R(s)$. On writing $s=\sigma+i t$ then, with $\sigma$ fixed, it follows that for large $t$

$$
\exp \{(D s-1 / 2) \log D s-D s\} \sin \pi(K-D s) / 2=O\left(|s|^{(2 D \sigma-1) / 2}\right) .
$$

Consequently

$$
\begin{aligned}
R(s) & =s^{(1-D) / 2} O\left(|s|^{(2 D \sigma-1) / 2}\right) O\left(|s|^{-(2 r+1) / 2}\right) \\
& =O\left(|s|^{(2 D \sigma-D-2 r-1) / 2}\right) .
\end{aligned}
$$

Since $R(s)$ denotes the left hand side of (100a) it follows that we have established the lemma for the case when $(q-p)$ is odd. The proof for the case when $(q-p)$ is even is almost identical with the above investigation.

This lemma enables us to obtain the trigonometrical terms in the asymptotic expansion of $H(x)$ as in (87) for $(q-p)$ odd or (89) when $(q-p)$ is even. The successful application of this lemma to obtaining the asymptotic expansion of $H(x)$ is due to the way in which $Q(s)$ is expressed asymptotically in terms of Gamma functions as in (100a) and (100b). The idea of using forms such as $(100 \mathrm{a})$ or $(100 \mathrm{~b})$ originates in my previous paper on Fourier 
transforms, Fox $[3$, p. 411] where it was also used to obtain the trigonometrical terms of an asymptotic expansion.

The last two lemmas deal with an integral which, expressed in terms of the residues of its integrand, gives us the desired asymptotic expansion of $H(x),(87)$ or $(89)$. When $(q-p)$ is odd this integral will be denoted by $I_{1}$ and when $(q-p)$ is even by $I_{2}$.

$$
\begin{gathered}
I_{1}=\frac{1}{2 \pi i} \int\left\{Q(s)-\sum_{i=0}^{r} \nu_{i} \Gamma\left(D s-i+\frac{1-D}{2}\right) \sin \frac{1}{2} \pi(K-D s)\right\} \\
\cdot\left(\frac{x}{\beta}\right)^{-8} d s
\end{gathered}
$$

when $(q-p)$ is odd and

$$
\begin{gathered}
I_{2}=\frac{1}{2 \pi i} \int\left\{Q(s)-\sum_{i=0}^{r} \nu_{i} \Gamma\left(D s-i+\frac{1-D}{2}\right) \cos \frac{1}{2} \pi(K-D s)\right\} \\
\cdot\left(\frac{x}{\beta}\right)^{-8} d s
\end{gathered}
$$

when $(q-p)$ is even. We now have two aims, the first to show that if the path of integration of $I_{1}$ or $I_{2}$ is the straight line $\sigma=\sigma_{0}$ then $I_{1}$ and $I_{2}$ are $O\left(x^{-\sigma_{0}}\right)$ and the second to show that this line may be completed into a closed contour by means of a large circular arc on the left of it. The residues inside this contour will then give us the desired asymptotic expansion (87) or (89).

LemMA 7. Assumptions: $s=\sigma+i t$; the path of integration of $I_{1}$ and of $I_{2}$ is the straight line $\sigma=\sigma_{0}, \sigma_{0}>1 / 2 ; a$ positive integer $r$ is chosen so that

$$
-D\left(\sigma_{0}-1 / 2\right)+(r+1 / 2)>1 \text {; }
$$

constants $\nu_{i}, i=0,1, \cdots, r$, are chosen according to the method specified by Lemma 6; conclusions:

$$
I_{1}=O\left(x^{-\sigma_{0}}\right), \quad I_{2}=O\left(x^{-\sigma_{0}}\right) .
$$

To prove this we use Lemma $6,(100 a)$ or $(100 b)$, to conclude that the modulus of the integrand of either $I_{1}$ or $I_{2}$ is $O\left(|s|\left(-2 r-1+2 D \sigma_{0}-D\right) / 2\right)(x / \beta)^{-\sigma_{0}}$. Hence, from (104), as a function $s$ either of these integrands is $O\left(|s|^{-1-c}\right)$ where $\epsilon>0$ and so both integrals are absolutely convergent. Consequently, considered as a function of $x$, each integral must be $O\left(x^{-\sigma_{0}}\right)$. This completes the proof of Lemma 7.

LEMMA 8. The straight line $\sigma=\sigma_{0}$ divides the circle whose centre is the origin and whose radius is $\rho$ into two arcs. Let $A$ denote the arc on the left of $\sigma=\sigma_{0}$. Then, taken along the $\operatorname{arc} A, \lim _{\rho \rightarrow \infty} I_{1}=0$ and $\lim _{\rho \rightarrow \infty} I_{2}=0$.

We assume that as $\rho \rightarrow \infty$ the arc $A$ avoids crossing any pole of either of 
the integrands of $I_{1}$ or $I_{2}$. This is always possible since all these poles are simple and isolated.

To prove this let $(\rho, \theta),-\pi<\theta \leqq \pi$, be the polar co-ordinates of any point on the $\operatorname{arc} A$. On writing $s=\rho e^{i \theta}$ in the asymptotic expansion of $\Gamma(s+a),(92)$, it follows that for $|\theta| \neq \pi / 2,|\theta|<\pi-\delta, \delta>0$, the dominant factor is $\exp \{(\rho \log \rho) \cos \theta\}$. The case $|\theta-\pi|<\delta$ can be dealt with by using the formula $[7, \mathrm{p} .233] \Gamma(s+a)=\pi / \Gamma(1-s-a) \sin \pi(s+a)$ and for such values of $\theta$ it is found that the dominating factor is the same.

On applying this result to the various terms of the integrands of $I_{1}$ and $I_{2}$, then, for points $(\rho, \theta)$ and $|\theta| \neq \pi / 2$ we find that the dominant factor in each case is $\exp \{(D \rho \log \rho) \cos \theta\}$. Since $D>0$ we then deduce that if the arc of integration is confined to those parts of $A$ for which $|\theta|>\pi / 2$, then $\lim I_{1}=0$ and $\lim I_{2}=0$ as $\rho \rightarrow \infty$.

Again when $|\theta|=\pi / 2$ the dominant factor of $\Gamma(s+a)$ is

$$
s^{(2 a-1) / 2} \exp \{-s \pi / 2\} \text {. }
$$

Applying this to the various terms of the integrands of $I_{1}$ and $I_{2}$ it follows that for points $(\rho, \pm \pi / 2)$ the dominant factor for $Q(s)$ is $\rho^{-D / 2}$ and for either $\Gamma(D s-i+(1-D / 2) \sin \pi(K-D s) / 2$ or $\Gamma(D s-i+(1-D) / 2) \cos \pi(K-D s) / 2$ the dominating factor is $\rho^{-(2 i+D) / 2}$. Hence, when $|\theta|=\pi / 2$, these integrands tend to zero as $\rho \rightarrow \infty$. Jordan's theorem $[7$, p. 115] therefore justifies an extension of the result just above to the end points so that we have

$$
\lim _{\rho \rightarrow \infty} I_{1}=0 \quad \text { and } \quad \lim _{\rho \rightarrow \infty} I_{2}=0
$$

where the path of integration in each case is the semicircular part of $A$ for which $|\theta| \geqq \pi / 2$.

We still have to deal with the two arcs of $A$ which lie between the imaginary axis, where $|\theta|=\pi / 2$, and the line $\sigma=\sigma_{0}$. The total length of these two $\operatorname{arcs}$ is $2 \rho \operatorname{arc} \sin \left(\sigma_{0} / \rho\right)=O(1)$ as $\rho \rightarrow \infty$.

The order of the integrands of $I_{1}$ and $I_{2}$ along these arcs can be estimated from Lemma 6. Assuming that the constants $\nu_{i}, i=0,1, \cdots, r$, have been chosen in the manner specified in Lemma 6 then from (100a) and (100b) the order of the integrands is

$$
O\left(\rho^{(-2 r-1+2 D \sigma-D) / 2}\right)
$$

where $0 \leqq \sigma \leqq \sigma_{0}$. But on making use of the inequality (104) we have $\left(-2 r-1+2 D \sigma_{0}-D\right) / 2<-1$. Hence $(107)$ cannot exceed $O\left(\rho^{-1}\right)$.

Since the length of arc times the order of the integrand is $O\left(\rho^{-1}\right)$ it follows that when taken along the two arcs of $A$ lying between the lines $\sigma=0$ and $\sigma=\sigma_{0}$ we have

$$
\lim _{\rho \rightarrow \infty} I_{1}=0 \text { and } \quad \lim _{\rho \rightarrow \infty} I_{2}=0
$$


The results (106) and (108) between them evidently give a complete proof of Lemma 8 . We can now proceed directly to the proof of the asymptotic expansion of $H(x)$.

12. The asymptotic expansion of $H(x),(x>0)$. We can now find the asymptotic expansion of $H(x)$ with the prescribed error term $O\left(x^{-\sigma_{0}}\right)$. We shall assume that $\sigma_{0}>1 / 2$. The theory centres round the two integrals $I_{1}$ and $I_{2}$ of (102) and (103) one dealing with the case when $(q-p)$ is odd and the other when $(q-p)$ is even. The proofs are exactly the same, however, in both cases and so we shall confine ourselves to the case when $(q-p)$ is odd. The integral we must study is then (102) which we repeat,

$$
\begin{gathered}
I_{1}=\frac{1}{2 \pi i} \int_{\sigma_{0}}\left\{Q(s)-\sum_{i=0}^{r} \nu_{i} \Gamma\left(D s-i+\frac{1-D}{2}\right) \sin \frac{1}{2} \pi(K-D s)\right\} \\
\cdot\left(\frac{x}{\beta}\right)^{-s} d s
\end{gathered}
$$

where the path of integration is the straight line $\sigma=\sigma_{0}$, and $Q(s)$ is as defined in (91).

We assume temporarily that the path of integration does not cross any pole of the integrand of $I_{1}$. Since $\sigma_{0}>1 / 2$ this means that $\sigma_{0}$ cannot take any of the values $\left\{\operatorname{Re}\left(a_{n}\right)+i\right\} / e_{n}, n=1, \cdots, p ; i=0,1,2, \cdots$. We shall at the end of this section show that even if $\sigma_{0}$ does assume one of these values the asymptotic expansions (87) and (89) still remain true.

Given $\sigma_{0}$ we now choose $r$ to be the greatest positive integer in $D\left(\sigma_{0}-1 / 2\right)$ $+3 / 2$ so that we have

$$
1<-D\left(\sigma_{0}-1 / 2\right)+r+1 / 2<2 .
$$

The left hand inequality of (110) is the same as the inequality (104) and therefore, if the constants $\nu_{i}, i=0,1,2, \cdots, r$, are chosen in the manner specified by Lemma 6 , according to Lemma 7 we have $I_{1}=O\left(x^{-\sigma_{0}}\right)$. We assume, of course, that these constants are so chosen.

From Lemma 8 we know that the straight line path $\sigma=\sigma_{0}$ of (109) can be completed to a closed contour by means of a large circular arc to the left, an arc whose centre is the origin and whose radius tends to infinity. Hence $I_{1}$ is also equal to the sum of all the residues of the integrand of (109) lying to the left of the line $\sigma=\sigma_{0}$. As a final conclusion we can state that the sum of all the residues of (109) due to the poles of the integrand on the left of $\sigma=\sigma_{0}$ is $O\left(x^{-\sigma_{0}}\right)$.

We now proceed to compute all these residues to the left of $\sigma=\sigma_{0}$ and for convenience we shall place them in three groups.

Group 1 contains all the residues of $Q(s)(x / \beta)^{-s}$ which arise from the poles of $\Gamma\left(b_{m}+c_{m} s\right), m=1, \cdots, q$. All these poles are to the left of $\sigma=\sigma_{0}(>1 / 2)$. In fact from condition (ii) of Theorem 9 they all lie to the left of $\sigma=0$. Evidently these residues are the same as those of the integral in (90) which 
defines $H(x)$. Hence the sum of the residues in this group is $H(x)$.

Group 2 contains all the residues of $Q(s)(x / \beta)^{-*}$ which arise from those poles of $\Gamma\left(a_{n}-e_{n} s\right), n=1, \cdots, p$, lying to the left of $\sigma=\sigma_{0}$. According to condition (ii) of Theorem 9 all these poles lie to the right of the line $\sigma=1 / 2$ so that all the poles of this group lie between the lines $\sigma=1 / 2$ and $\sigma=\sigma_{0}(>1 / 2)$.

If $u_{n}$ denotes the greatest positive integer in $e_{n} \sigma_{0}-\operatorname{Re}\left(a_{n}\right)$ then the poles of this group are at $s=\left(a_{n}+i\right) / e_{n}$, where $i=0,1,2, \cdots, u_{n}$ and $n=1,2, \cdots, p$. The corresponding residues are then

$$
-\sum_{n=1}^{p}\left\{x^{-a_{n} / e_{n}}\left(A_{n}+B_{n} x^{-1 / e_{n}}+C_{n} x^{-2 / e_{n}}+\cdots+U_{n} x^{-u_{n} / e_{n}}\right)\right\},
$$

where $A_{n}, B_{n}, C_{n}, \cdots, U_{n}$ are respectively the residues of $-Q(s) \beta^{s}$ at the poles $s=\left(a_{n}+i\right) / e_{n}, i=0,1,2, \cdots, u_{n}$. Since $Q(s)$ is a ratio of two products of Gamma functions, (91), these residues are easy to compute and consequently $A_{n}, B_{n}, \cdots, U_{n}$ are all easy to compute in comparison with the constants $\nu_{i}, i=0,1, \cdots, r$, of Lemma 6 , which may be very difficult to compute.

The expression (111) gives us all the algebraic terms of the asymptotic expansion of $H(x)$ as required by (87) or (89) in the statement of Theorem 9. The constants $A_{n}, B_{n}, C_{n}, \cdots, U_{n}$ of (87) and (89) are the same as those of (111). The statement of Theorem 9 contains the phrase "the constants $A_{n}, B_{n}, C_{n}, \cdots, U_{n}, n=1, \cdots, p$, are computed by means of residues as specified in $\$ 12$." The method of computation referred to in this phrase is the one immediately following expression (111).

Groups 1 and 2 between them account for all the residues of $Q(s)(x / \beta)^{-8}$.

Group 3 contains all the residues of

$$
-\left\{\sum_{i=0}^{r} \nu_{i} \Gamma\left(D s-i+\frac{1-D}{2}\right) \sin \frac{1}{2} \pi(K-D s)\right\}\left(\frac{x}{\beta}\right)^{-8}
$$

arising from the poles of this expression which lie to the left of the line $\sigma=\sigma_{0}$. All the poles of (112) are simple and isolated and occur when $s=\{i / D-(1-D) / 2 D-j / D\}$, where $i=0,1,2, \cdots, r$ and $j=0,1,2, \cdots$. The pole at the extreme right of this set of points occurs when $i=r$ and $j=0$. The extreme right pole is at

$$
s=\frac{1}{D}\left(r+\frac{D+1}{2}-1\right)>\sigma_{0},
$$

by the left hand inequality of (110). Hence this pole is outside the contour. The pole immediately on the left of (113) occurs when $i=r$ and $j=1$ or when $i=(r-1)$ and $j=0$. This pole is at

$$
s=\frac{1}{D}\left(r+\frac{D+1}{2}-2\right)<\sigma_{0},
$$


by the right hand inequality of (110). Consequently, of all the poles of (112) one is to the right of the line $\sigma=\sigma_{0}$ and so is outside our contour while all the others are to the left of this line and their residues must all be included when evaluating $I_{1}$ of (109).

The residue due to the pole (113), which is outside the contour, is evidently a constant multiple of $x^{-s}$ where, by (113), $s>\sigma_{0}$. Hence this residue cannot exceed $O\left(x^{-\sigma_{0}}\right)$, so that if this residue is added to the others we shall only include a magnitude which is of the same order as the error term we were allowed at the beginning of this section. This justifies us in dealing with (112) as if all its poles lie to the left of the line $\sigma=\sigma_{0}$, i.e., in including the pole (113).

The residues of (112) are easily found to be

$$
-\left(\frac{x}{\beta}\right)^{(1-D) / 2 D} \sum_{i=0}^{r} \nu_{i}\left(\frac{x}{\beta}\right)^{-i / D} \sin \left\{\frac{1}{2} \pi\left(K-i+\frac{1-D}{2}\right)+\left(\frac{x}{\beta}\right)^{1 / D}\right\} .
$$

These are all the trigonometrical terms of (87) or (89).

The sum of the residues in these three groups is, by Cauchy's theorem, equal to the value of the integral of (109) and this, in turn, we know to be $O\left(x^{-\sigma_{0}}\right)$ by Lemma $7,(105)$. On comparing these statements with (87) we see that the asymptotic expansion for $H(x)$ has been proved for the case when $(q-p)$ is an odd positive integer.

The case when $(q-p)$ is an even positive integer is dealt with in the same way; we must use $I_{2}$ of (103) instead of $I_{1}$ of (109). We then obtain (89) as the asymptotic expansion of $H(x)$.

We conclude this section by reviewing one point which was mentioned at the beginning. It was assumed that the path of integration of $I_{1}$, of (109), did not pass through any of the poles of the integrand of $I_{1}$.

Suppose now that $\sigma_{0}$ is prescribed in such a manner that the path of integration of $I_{1}$ does pass through a pole, $P$ say, of the integrand. In this case we must insert a small semicircular indentation in the line $\sigma=\sigma_{0}$, with $P$ as centre, and drawn so that $P$ is either inside or outside the modified contour. In either case on making the radius of the indentation tend to zero it is easy to show that the effect of the indentation is to introduce a term of order $O\left(x^{-\sigma_{0}}\right)$. Consequently the asymptotic expansions (87) and (89) still remain true even if $\sigma_{0}$ takes any of the values $\left\{\operatorname{Re}\left(a_{n}\right)+i\right\} / e_{n}, n=1,2, \cdots, p$ and $i=0,1,2, \cdots$.

This completes the proof of Theorem 9.

13. Some properties of $H(x)$. The asymptotic expansions, (87) and (89), for $H(x)$ contain as a special case a theorem I have proved in a previous paper [3, Lemma 1, p. 409]. The previous result can be obtained from (87) by writing $c_{m}=e_{n}=c, m=1, \cdots, q, n=1, \cdots, p$ and $q=p+1$. The method used to prove the previous result, however, is the same as the one used here. 
Both methods depend upon the introduction and study of expressions such as (100a) and (100b) used here.

The asymptotic expansions for $H(x),(87)$ and (89) also contain the asymptotic expansion given in (35) as a special case since the $G(x)$ function of (10) can be obtained from $H(x)$ of (52) by writing $c_{m}=e_{n}=c, m=1, \cdots, q$, $n=1, \cdots, p$, in (52). Our proof, however, restricts $x$ to be real and positive, whereas the work of Barnes [1] and Meijer [4] on the $G(x)$ function allows $x$ to be complex.

Finally the asymptotic expansion of $H(x)$ enables us to explain the need for the transformations (66) which replace $H(x)$ by another kernel $H\left\{(X \alpha)^{D}\right\}(X \alpha)^{(D-1) / 2} D \alpha^{1 / 2}$.

From condition (ii) Theorem 7 we see that the algebraic terms of the asymptotic expansion for $H(x),(87)$ and (89), tend to zero as $x \rightarrow \infty$. But the trigonometrical terms contain the factor $x^{(1-D) / 2 D}$. Hence, when $D \neq 1, H(x)$ cannot oscillate finitely, as $x \rightarrow \infty$, in the manner of the classical Fourier kernels.

On the other hand (87) and (89) show that the asymptotic expansion of $H\left\{(X \alpha)^{D}\right\}(X \alpha)^{(D-1) / 2} D \alpha^{1 / 2}$ contains algebraic terms which tend to zero as $x \rightarrow \infty$, but that the trigonometrical terms are of the type

$$
\sum_{i=0}^{r} \nu_{i} X^{-i} \sin \left\{\frac{1}{2} \pi\left(K-i+\frac{1-D}{2}\right)+X\right\} \text {. }
$$

Evidently the terms of (116) for which $i>1$ all tend to zero as $X$ tends to infinity but the term for which $i=0$ oscillates finitely as $X \rightarrow \infty$. Hence this kernel behaves much like the symmetrical Fourier kernels of classical theory and so much of this theory can be applied to it. This explains the need for the transformations (66) and the method of proof used in the establishment of Theorem 7 .

\section{REFERENCES}

1. E. W. Barnes, The asymptotic expansion of integral functions defined by generalized hypergeometric series, Proc. London Math. Soc. Ser. 2 vol. 5 (1907) pp. 59-117.

2. The Bateman Manuscript Project, Higher transcendental functions, Vol. 1, New York, McGraw-Hill Book Co., 1953.

3. C. Fox, A generalization of the Fourier Bessel integral transform, Proc. London Math. Soc. Ser. 2 vol. 29 (1929) pp. 401-452.

4. C. S. Meijer, On the G-function, Proc. Nederl. Akad. Wetensch. vol. 49 (1946) pp. 227237, 344-356, 457-469, 632-641, 765-772, 936-943, 1062-1072, 1165-1175.

5. Roop Narain, A Fourier kernel, Math. Z. vol. 70 (1959) pp. 297-299.

6. E. C. Titchmarsh, Introduction to the theory of Fourier integrals, Oxford, University Press, 1937.

7. E. T. Whittaker and G. N. Watson, $A$ course of modern analysis, Cambridge, University Press, 1915.

MCGILl UNIVERSITY, Montreal, Canada 